\title{
Mode Shifting Between Storage and Recall Based on Novelty Detection in Oscillating Hippocampal Circuits
}

\author{
M. Meeter, ${ }^{*}$ J.M.J. Murre, and L.M. Talamini
}

\begin{abstract}
It has been suggested that hippocampal mode shifting between a storage and a retrieval state might be under the control of acetylcholine (ACh) levels, as set by an autoregulatory hippocampo-septohippocampal loop. The present study investigates how such a mechanism might operate in a large-scale connectionist model of this circuitry that takes into account the major hippocampal subdivisions, oscillatory population dynamics and the time scale on which ACh exerts its effects in the hippocampus. The model assumes that hippocampal mode shifting is regulated by a novelty signal generated in the hippocampus. The simulations suggest that this signal originates in the dentate. Novel patterns presented to this structure lead to brief periods of depressed firing in the hippocampal circuitry. During these periods, an inhibitory influence of the hippocampus on the septum is lifted, leading to increased firing of cholinergic neurons. The resulting increase in ACh release in the hippocampus produces network dynamics that favor learning over retrieval. Resumption of activity in the hippocampus leads to the reinstatement of inhibition. Despite theta-locked rhythmic firing of ACh neurons in the septum, ACh modulation in the model fluctuates smoothly on a time scale of seconds. It is shown that this is compatible with the time scale on which memory processes take place. A number of strong predictions regarding memory function are derived from the model. $\odot 2004$ Wiley-Liss, Inc.
\end{abstract}

KEY WORDS: acetylcholine; computational modeling; hippocampus; medial septum; memory

\section{INTRODUCTION}

Over the past decades, the hippocampus has been implicated in various functions, including episodic memory (Squire, 1992; Eichenbaum et al., 1999) and novelty detection of the contextual or spatial aspects of an experience (Johnson and Moberg, 1980; Kitchigina et al., 1997; Zhu et al., 1997; Knight and Nakada, 1998; Xiang and Brown, 1998; Montag-Sallaz et al., 1999; Lisman and Otmakhova, 2001; Mumby et al., 2002). From existing experimental studies, it is difficult to understand how these functions are interrelated, and whether they are subserved by the same processes or by different ones at the circuit level.

Modeling studies have proposed that hippocampal novelty detection may regulate levels of acetylcholine (ACh), which, in turn, are involved in shifting hippocampal dynamics to favor either encoding or retrieval (Hasselmo et al., 1995). The mechanism was suggested in view of various data regarding the effects of ACh on memory tasks and hippocampal physiology, and also because neural network requirements for learning and for retrieval are not

Department of Psychology, University of Amsterdam, Amsterdam, The Netherlands

*Correspondence to: M. Meeter, Department of Cognitive Psychology, Vrije Universiteit Amsterdam, Vd Boechortstraat 1, 1081 BT Amsterdam, The Netherlands. E-mail: m@meeter.nl

Accepted for publication 13 October 2003

DOI 10.1002/hipo.10214

Published online 10 March 2004 in Wiley InterScience (www.interscience. wiley.com). compatible in hippocampus-type circuits (Murre, 1992; Hasselmo, 1995; Hasselmo et al., 1996). With the proposed mechanism, information with high novelty content would induce hippocampal dynamics that favor learning of the input, while input that is similar to already stored patterns would induce a state that enhances retrieval of related information. Thus, the system would preferentially encode novel stimuli in a learning mode, whereas during retrieval the animal could generate predictions regarding the situation at hand. Moreover, little learning would take place during retrieval so that existing patterns are protected from modification.

The validity of this hypothesis has been questioned in view of evidence suggesting that ACh may exert a more sustained influence on hippocampal activity than the aforementioned models assume. Indeed, both the depolarization of pyramidal cells (Cole and Nicoll, 1984; Stewart and Fox, 1989a; Hasselmo and Fehlau, 2001) and dampening of transmission in Schaffer collaterals by ACh (Hasselmo and Fehlau, 2001) may develop slowly, during the course of a few seconds after ACh release, and may then last $10 \mathrm{~s}$ or more. This has led some investigators to conclude that, although ACh may have a role in shifts over longer intervals (e.g., between learning and consolidation), its dynamics are too slow to underlie mode shifting between encoding and retrieval, which is assumed to occur on the time scale of tens or hundreds of milliseconds (Hasselmo and Fehlau, 2001; Hasselmo et al., 2002). Hasselmo and colleagues propose that other substances may set dynamics on a scale fast enough for the system to switch between learning and retrieval mode.

However, considering the time scale at which natural learning and retrieval take place, slow cholinergic modulation may well be appropriate for regulation of mode shifts. For instance, judging from their exploratory responses, it takes rats many minutes to familiarize themselves with a novel environment, and at least many seconds to a few minutes to explore known objects in locations where they have not previously encountered them (Mumby et al., 2002). Even stronger evidence comes from contextual fear conditioning: rats will only associate a shock to a new context if they have had many seconds to form a representation of that context (Fanselow, 2000). In fact, if rats are shocked before they have developed a representation of their surroundings, fear can even be conditioned to the memory representation of a different context when the right cues are present 
(Rudy et al., 2002). In experiments with human subjects, study times tend to have small effects on retrieval rates when they are more than $2 \mathrm{~s}$ per list item. However, with shorter study times, long-term recall deteriorates (Roberts, 1972; Murdock, 1974). This is not a result of failing perceptual processing, as visual scenes can be processed at speeds of up to 8 per second (Potter, 1976). The storage of context information may be one factor in determining the effect of learning times on subsequent retrieval; recently, it has been suggested that this may take up to $2 \mathrm{~s}$ (Malmberg and Shiffrin, 2003). Such contextual, or configurative, aspects of learning putatively depend on the hippocampus (Myers and Gluck, 1994; Wan et al., 1999).

In view of the above, the present study reinvestigates the ACh account of mode shifting in a large-scale model of the hippocampal formation and basal forebrain, featuring the entorhinal cortex (EC), dentate gyrus (DG), CA3, CA1, and the medial septum. The model incorporates many features of this circuitry, including structural and functional properties of the subregions and connections, feedback and feedforward inhibition, and oscillatory population dynamics in the theta $(4-10 \mathrm{~Hz})$ and gamma $(20-40 \mathrm{~Hz})$ frequency range (Shimono et al., 2000). In addition, most of the known effects of ACh at the cellular level were modeled, with realistic onset and decay time constants for the effects of ACh.

The biological detail in the model places strong constraints on the mechanisms underlying novelty detection and memory function, and allows differential contributions of various network components to be distinguished. Furthermore, the model is to some extent explicit with regard to the timing of events in the circuitry which allows the investigation of temporal aspects of memory processing; for instance, the duration of the novelty signal, the time course of mode shifting, and the approximate time it may take for the hippocampus to form a representation. Simulations explore how these processes interact, and whether they lead to plausible memory performance, in accordance with the time scale at which natural learning takes place.

The next section presents the model. First, the different modules-corresponding to areas of the hippocampal formation-are discussed with their incoming and outgoing connections. The implementation of oscillatory population activity and ACh effects are then described. Later sections of this article present simulations that illustrate how the model learns and retrieves, and how it switches between these functions. Technical details have been relegated to the Appendix.

\section{THE MODEL}

\section{Modules and Connectivity}

The hippocampus has been the focus of much theorizing and many modeling efforts (O'Keefe and Nadel, 1978; O'Reilly and McClelland, 1994; McClelland et al., 1995; Squire and Alvarez, 1995; Hasselmo et al., 1996; Rolls, 1996; Eichenbaum et al., 1999; Lisman, 1999; Nadel et al., 2000; Suzuki, 2000; Meeter et al., 2002). Out of this work, a common architecture has emerged, capturing the most prominent features of the hippocampal system.

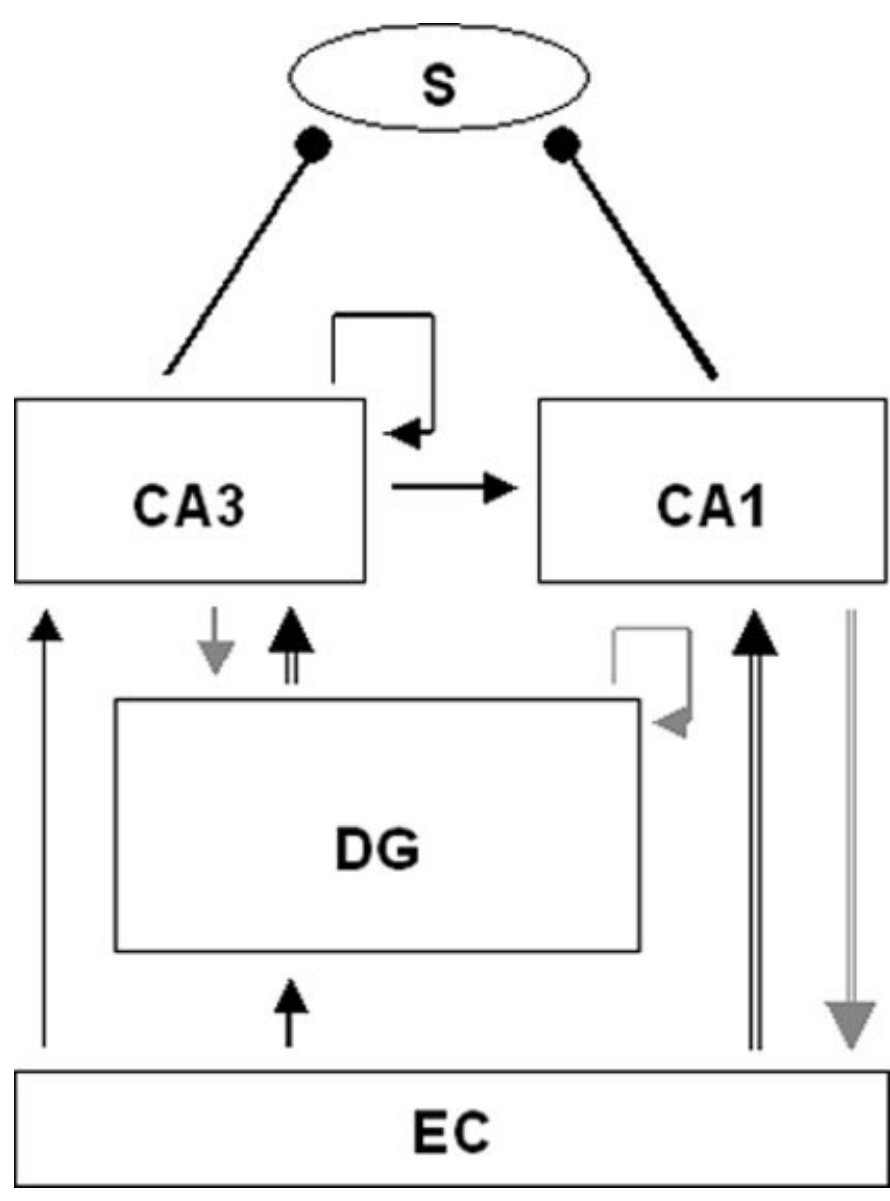

FIGURE 1. Drawing of all hippocampal areas and connections in the model. Gray arrows depict connections that are known to exist, but were not modeled. Solid connections are fanning, while open connections are point-to-point. Connections ending with a circle are inhibitory.

The architecture of the present model incorporates many of these features, while adding new ones.

The model was built using spiking McGregor model neurons. These integrate-and-fire nodes were abstracted from the HodgkinHuxley formalism and incorporate sodium, potassium, and chloride currents, implementing excitatory and inhibitory inputs, leak currents, and adaptation (MacGregor and Oliver, 1974). Hebbian learning was used, with the addition of negative Hebbian learning that models LTD. As the unit of time in our model, we chose $2 \mathrm{~ms}$ per time step. Formal descriptions of the model neuron, inhibition, and learning are given in the Appendix.

The model consists of four layers representing subfields of the hippocampal formation: the EC, the granule layer of the DG, CA3, and CA1. The connectivity between the model components (Fig. 1) reflects the existing pathways in the hippocampus (for review, see Witter et al., 2000). Some known connections were not included in the model-notably those from CA3 back to the DG (Scharfman, 1996).

Parameter settings of the model were based on neurobiological data, where available. Table 1 lists several layer parameters, as well as data for rat hippocampus, from which the model parameters 
TABLE 1.

Values for Layer Parameters*

\begin{tabular}{|c|c|c|c|c|}
\hline & $\mathrm{EC}$ & DG & CA3 & CA1 \\
\hline \multicolumn{5}{|c|}{ No. of neurons } \\
\hline Rat & $200,000^{c, d}$ & $1,000,000^{\mathrm{c}} ; 850,000^{\mathrm{d}}$ & $125,000^{\mathrm{c}} ; 160,000^{\mathrm{d}}$ & $316,000^{c} ; 250,000^{d}$ \\
\hline Model & 80 & 240 & 60 & 100 \\
\hline \multicolumn{5}{|c|}{ Proportion of active neurons ${ }^{a}$} \\
\hline Rat & $6.25 \%{ }^{\mathrm{d}}$ & $0.39 \%{ }^{\mathrm{d}}$ & $2.42 \%^{\mathrm{d}}$ & - \\
\hline Model & $12 / 80$ & $10 / 240$ & $10 / 60$ & $12 / 80$ \\
\hline \multicolumn{5}{|l|}{$\beta^{\mathrm{b}}$} \\
\hline Model & 0.5 & 2 & 0.5 & 0.5 \\
\hline
\end{tabular}

EC, entorhinal cortex; DG, dentate gyrus.

*For comparison, published data about similar parameters in the rat are given for the number of neurons and activity in subregions of the hippocampus

a Proportion of active neurons, maximal activity in each layer.

${ }^{b}$ Feedback inhibition parameter (see Equation 8 in the Appendix).

${ }^{c}$ Amaral et al. (1990) (average of values in their Table 1).

dO'Reilly and McClelland (1994).

were derived. Table 2 presents the parameter values for the connections in the model, including density, organization and relative strength. Justification for these values is given below.

The EC is the main cortical input structure of the hippocampus (Witter et al., 2000). In reality, layers II and III of this structure project to different subdivisions of the hippocampus proper but, as the current model is mostly concerned with intrahippocampal processing, one single $\mathrm{EC}$ input layer propagates the same information to DG, CA3, and CA1. The deep layers of the EC, which receive hippocampal output, were not implemented.

To avoid unwarranted assumptions concerning the input to the EC, we used a constant input of medium strength, activated by clamping a single "lower level" node connected to a random subset of EC nodes via uniform weights. In line with anatomical findings (Witter et al., 2000), the EC projections to the DG and CA3 are random (projection field $40 \%$ ), while the projection to CA1 is topologically organized (point-to-point).

The next layer in the circuit is the DG. This module contains a high number of dentate granule cells, few of which fire at any given moment (Table 1). The resulting sparseness of activation, as well as the fanning input projection from the EC, are considered a prerequisite for orthogonalization (Marr, 1971). Orthogonalization implies that patterns that are correlated in a given layer generate uncorrelated representations in the projection field. This is often

TABLE 2.

Parameter Values for the Different Connections in the Network

\begin{tabular}{lcccccc}
\hline & EC-DG $^{\mathrm{a}}$ & EC-CA3 & DG-CA3 & CA3-CA3 & CA3-CA1 & EC-CA1 \\
\hline Density $^{\mathrm{b}}$ & $40 \%$ & $40 \%$ & 3 from each Dg-node & $75 \%$ & $75 \%$ & 1 per node \\
Initial weight strength $^{\mathrm{c}}$ & 0.09 & 0.06 & 1 & 0.06 & 0.08 & 0.4 \\
Maximum weight $^{\mathrm{d}}$ & 0.18 & 0.12 & 1 & 0.12 & 0.2 & 0.4 \\
$\mu^{\mathrm{c}}$ & $0.04^{*} \Psi$ & 0.02 & 0 & $0.05^{*} \Psi$ & $0.05^{*} \Psi$ & 0 \\
Dampening by ACh $^{\mathrm{f}}$ & - & - & - & $1-0.5^{*} \Psi$ & $1-0.5^{*} \Psi$ & - \\
Feedforward inhibition $^{\mathrm{g}}$ & 0.15 & 0.15 & 0.25 & & 0.15 & 0.2 \\
\hline
\end{tabular}

EC-DG, entorhinal cortex-dental gyrus; ACh, acetylcholine.

${ }^{a} \mathrm{EC}-\mathrm{DG}$, connection from EC to DG.

${ }^{b}$ Number of postsynaptic target nodes for each presynaptic node (absolute or as percentage from the layer) targeted by any presynaptic node.

'Initial weight strength, weight at the start of simulation.

${ }^{\mathrm{d}}$ Maximum weight, maximum attainable weight of any connection.

e Learning rate.

${ }^{\mathrm{f}}$ Dampening of transmission under influence of acetylcholine.

${ }^{\mathrm{g}}$ Feedforward inhibition, strength of this form of inhibition ( $\lambda$ in Equation 8 of the Appendix). 
conceptualized to be an important contribution of the DG to hippocampal processing (McNaughton and Morris, 1987; O’Reilly and McClelland, 1994). Although orthogonalization results from divergent projections and sparse firing alone (Marr, 1971), it is strengthened by long-term potentiation (LTP) and long-term depression (LTD), both of which were implemented in the EC to DG connection (O'Reilly and McClelland, 1994).

The DG nodes send powerful point-to-point excitation to the CA3 layer, which is not modulated by Hebbian learning and is accompanied by strong feedforward inhibition (Acsády et al., 1998; Henze et al., 2000). DG principal cells tend to have relatively extended refractory periods, but they are capable of firing at around gamma frequency, as they do in the model (Henze et al., 2000). Mossy cells, another type of excitatory cell in the dentate, were not implemented in the model.

CA3 receives inputs from the EC cortex and from the DG; it sends strong, fanning, projections to CA1 via the Schaffer Collaterals (Witter et al., 2000). In the literature, it has been suggested that CA3 may be involved in either autoassociative learning and pattern completion (Marr, 1971; McNaughton and Morris, 1987; Treves and Rolls, 1994) or heteroassociative learning and sequence recall (O’Keefe and Recce, 1993; Levy, 1996; Skaggs et al., 1996; Lisman, 1999). Both proposals are motivated by the existence of extensive recurrent connections among CA3 principal cells (Amaral et al., 1990; Li et al., 1994), but they differ in the time scale on which learning is proposed to take place (learning over short intervals - within a gamma period-would lead to autoassociation, while learning over longer intervals would enable heteroassociation). In the model, CA3 is the only layer to have internal connectivity, with each node being connected to a random $40 \%$ of the other nodes. For the sake of simplicity, CA3 collaterals in the present model only have a weak autoassociative role. It is expected, however, that the proposed regulatory mechanism would also function under the constraints posed by heteroassociative memory function.

The CA1 region of the hippocampus receives a direct projection from the EC via the perforant path (Yeckel and Berger, 1990), and an indirect one, via the so-called tri-synaptic loop (the pathway over DG and CA3). Both projections are incorporated in the model. CA1 has been proposed to function as a translator between the code of CA3 and the cortical code (McClelland and Goddard, 1996). In the present model, CA1 associates the pattern in CA3 with the pattern in EC. In case of a high ACh level, transmission through the Schaffer collaterals is dampened and activity in CA1 is dominated by the EC. This allows the CA3-CA1 connection to store the association between the EC pattern and the CA3 pattern. In contrast, with low ACh levels, the influence of Schaffer collateral input in CA1 is relatively larger, and is the major determinant of which nodes are activated in CA1. The function of CA1 in those instances is that of relaying the reinstated CA3 pattern to the EC and to other output structures. As the reciprocal connections between EC layer III and CA1 are topologically organized, CA1 neurons innervated by particular EC layer III neurons may be able to reactivate these neurons during retrieval. The connections from CA1 to the deep EC layers might also contribute to this "closing of the loop." This would complete the transfer of information back to neocortical areas. In the current version of the model, however, output of CA1 to either layer III or the deep EC layers is not implemented.

The above rationale requires that $\mathrm{EC}$ inputs determine which nodes fire in CA1 during learning. However, evidence suggests that the EC-CA1 pathway has a large inhibitory component and does not trigger CA1 pyramidal neurons by itself in anaesthetized rats (Canning et al., 2000). Moreover, if CA1 nodes would be made to fire by EC inputs alone, they would likely fire before CA3 nodes, which might, in view of the temporal asymmetry in realbrain Hebbian learning, lead to LTD instead of LTP in the Schaffer collaterals. A solution is that EC inputs lead to subthreshold depolarization in those CA1 cells targeted by the pattern in EC (Hasselmo and Wyble, 1997). As a new pattern in CA3 will send relatively nonspecific activation to CA1, these depolarized CA1 nodes will have a high likelihood to be brought to fire by Schaffer collateral inputs. While EC thus biases some CA1 nodes to fire, it is CA3 input that triggers them. Indeed, stimulation of CA3 in the rat hippocampus readily elicits firing in CA1 (Canning et al., 2000).

Activity in the model was controlled by fast modular inhibition provided by one, fully connected, inhibitory node for each layer (see Appendix). Hereby, the plausible assumption was made that all types of inhibition are relatively untargeted within a layer (Buhl et al., 1994; Cobb et al., 1995; Miles et al., 1996; Acsády et al., 1998). The inhibitory nodes did not have integrate-and-fire dynamics; instead these nodes emitted a continuous output, approximating the summed activity of a whole population of interneurons. The magnitude of the inhibitory signal they sent out depended on activity in the layer they were connected to (feedback inhibition), as well as on activity in modules projecting to this layer (feedforward inhibition). The feedforward inhibition was conveyed via excitation of the inhibitory node of the receiving layer (Wierenga, 2002). Each modeled pathway thus consisted of an excitatory and an inhibitory component, which were balanced in strength. The temporal patterning of inhibition was set to approximate fast $\gamma$-aminobutyric acid (GABA)-A channel mediated inhibition. To further control the network, there was a maximum " $\mathrm{k}$ " to the number of nodes that could fire in a layer at any given moment.

\section{Oscillatory Activity}

Oscillatory population dynamics, within the range of $4-12 \mathrm{~Hz}$ (theta frequency), occur in the hippocampus, septum, and various cortical regions of the brain during alert waking and rapid eye movement sleep (Green and Arduini, 1954; O'Keefe and Nadel, 1978). They have been suggested to set the temporal dynamics needed to organize synaptic plasticity and implement learning at the network level (Larson and Lynch, 1986; Huerta and Lisman, 1993; Hasselmo et al., 2002).

The medial septum-diagonal band complex is thought to entrain hippocampal cells to this rhythm, through a dual septo-hippocampal pathway. One component of this pathway originates from cholinergic septal cells, and targets both pyramidal and inhibitory cells in the hippocampal formation (Frotscher and Ler- 
anth, 1985). Although this projection enhances hippocampal and entorhinal theta (Stewart and Fox, 1990; Alonso and Klink, 1997; Tóth et al., 1997; Shimono et al., 2000), it is not crucial to its expression (Stewart and Fox, 1989a,b; Lee et al., 1999). The other component emanates from intrinsically rhythmic GABAergic neurons in the MS-DB. It targets a wide variety of nonpyramidal hippocampal neurons (Freund and Antal, 1988; Gulyás et al., 1990, 1991; Acsády et al., 1993) and phasically disinhibits them at theta frequency (Tóth et al., 1997). This results in concerted oscillations of hippocampal inhibitory cells, which induce rhythmic hyperpolarizations in the principal target cells and, in the background of tonic excitation by cholinergic and possibly other excitatory inputs, paces them into theta rhythm (Stewart and Fox, 1990; Ylinen et al., 1995).

Faster oscillations, at $20-100 \mathrm{~Hz}$, are also prominent in the hippocampal formation. In vivo, these so-called gamma rhythms occur during the theta state, superimposed on theta-waves, with interneuron firing entrained to both gamma and theta frequencies (Soltez and Deschenes, 1993; Bragin et al., 1995; Sik et al., 1995; Traub et al., 1998). The mechanisms generating these gamma oscillations involve networks of mutually inhibitory interneurons, likely basket and axo-axonic cells, that are excited by principal cells and ACh. These interneurons fire in gamma bursts, concomitant with increased principal cell activity, providing fast feedback inhibition to the perisomatic region of principal cells (Chrobak and Buzsaki, 1998; Traub et al., 1998; Shimono et al., 2000).

In the model, the inhibitory septal oscillator and a cholinergic node were simulated algorithmically (see Appendix). The septal oscillator phasically inhibits the ACh node and hippocampal inhibitory nodes, at theta frequency. The cholinergic node determines ACh levels in the hippocampal modules, as explained in the next section. In accordance with the aforementioned findings, gamma frequency oscillations in the model arise from an interaction of excitatory activity and fast feedback inhibition over the inhibitory nodes. In the simulations, this fast inhibitory feedback provides a powerful mechanism, limiting the recruitment of neurons into cell assemblies coding for system input.

\section{Acetylcholine and Learning Dynamics}

Many behavioral experiments suggest that $\mathrm{ACh}$ is necessary for episodic learning, while it is less important in retrieval (Ghoneim and Mewaldt, 1975; Peterson, 1977; Mewaldt and Ghoneim, 1979; Kopelman, 1986; Whishaw, 1989; Aigner et al., 1991). Most convincingly, a recent study showed that activation of the forebrain cholinergic pathways occurs during the acquisition of a rewarded operant response, while recall of the same behavior was not associated with the same activation of the cholinergic system (Orsetti et al., 1996). In support of the notion that ACh secretion is related to novelty, recent microdialysis studies show that during explorations of a new environment $\mathrm{ACh}$ levels are increased relative to baseline (Aloisi et al., 1997; Ceccarelli et al., 1999; Giovannini et al., 2001), while levels gradually decrease during consecutive explorations of the same test environment (Giovannini et al., 2001).
A hippocampal novelty signal could be relayed to the basal forebrain regions involved in hippocampal ACh secretion by hippocampo-septal fibers running in the fimbria. These fibers originate from a subpopulation of hippocampal inhibitory neurons, which are located mostly in stratum oriens of CA1 and CA3 (Alonso and Kohler, 1982; Tóth and Freund, 1992), and appear to target GABAergic septal projection neurons and ACh neurons (Tóth et al., 1993). These hippocampo-septal projection cells receive convergent input from large numbers of hippocampal principal cells (Tóth et al., 1993). Hippocampal activity may thus inhibit the septum; indeed, both fimbria stimulation (McLennan and Miller, 1974) and hippocampal sharp waves (Dragoi et al., 1999) were shown to inhibit neuronal activity in the medial septum.

In line with these findings, ACh release in the model is regulated by the summed activity in layers CA3 and CA1. This effect is obtained by subtracting the activity of the inhibitory nodes of the CA1 and CA3 layer from baseline activation of the septal cholinergic node. As a result, the amplitude of the theta band rhythmic activity of the ACh node decreases with increasing activity in CA3 and CA1. The formula governing septal activity contains a moving average implementing hypothesized slow kinetics in the inhibitory inputs from Ammon's horn to the septum. This is essential for novelty detection to work as envisioned, because only prolonged decreases in hippocampal activity (i.e., longer than one gamma cycle) indicate novelty. Rhythmic oscillations in hippocampal inhibition, particularly in the gamma range, are to large extent absorbed by this moving average, resulting in a relatively smooth fluctuation of ACh levels.

$\mathrm{ACh}$ release in the hippocampus is assumed to correspond, in linear fashion, with the activity of the cholinergic node. ACh release in turn determined the value of a variable symbolizing $\mathrm{ACh}$ modulation in the hippocampus. This latter variable determined all effects of ACh on several other variables in the model. Septal ACh release influenced ACh modulation via a dual exponential with onset and decay time constants obtained from Hasselmo and Fehlau (2001). The authors fitted these constants to their data on the time course of ACh modulation of excitatory synaptic potentials in slices of rat hippocampus. These time constants, together with slow kinetics in the inhibition of the septum, result in slowly changing ACh modulation in the model, in line with various experimental findings (Cole and Nicoll, 1984; Hasselmo and Fehlau, 2001).

As mentioned previously, substances that activate muscarinic receptors exert influences that are compatible with the mode-shifting hypothesis. The following effects of ACh were implemented as formulas modulating transmission, membrane kinetics and learning in various layers and connections of the model (formulas and parameter settings are given in the Appendix):

1. Preferential dampening of transmission over CA3 recurrent fibers and Schaffer collaterals to CA1 (Hounsgaard, 1978; Hasselmo and Schnell, 1994), through presynaptic inhibition

2. Slow, subthreshold depolarization of hippocampal principal cells, persisting for many seconds (Bernardo and Price, 1982; Cole and Nicoll, 1984; Caeser et al., 1993) 

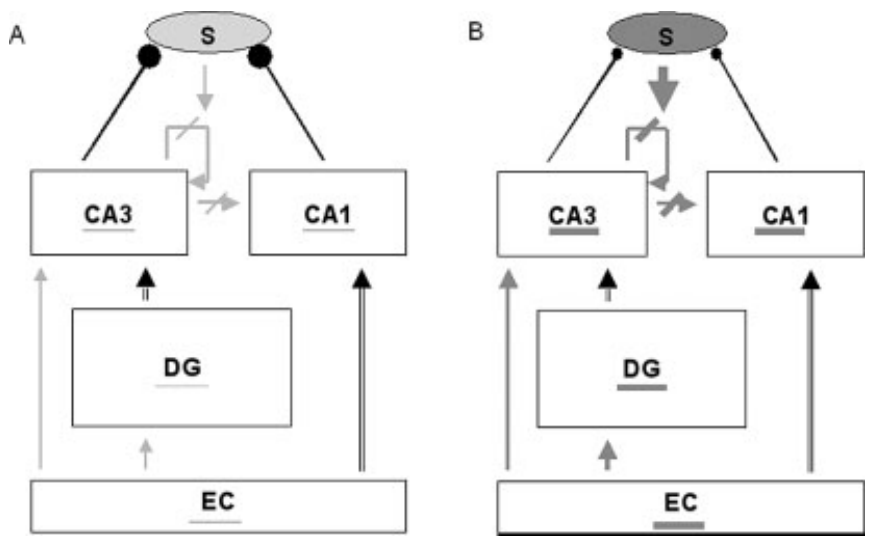

FIGURE 2. Functioning of the network in two modes: retrieval mode and learning mode. A: In retrieval mode, acetylcholine (ACh) modulation is relatively low. Thin gray lines indicate sites of $\mathrm{ACh}$ action in the system. B: In learning mode, ACh modulation is relatively high. As a consequence, LTP in several connections is boosted (dark arrows), while in some of these connections transmission is dampened (thick gray block). Several effects of ACh lead to easier and more prolonged firing in pyramidal cells of the hippocampus (thick gray underlining).

3. Enhancement of LTP at CA3 recurrent collateral synapses, at Schaffer collateral synapses in CA1 (Blitzer et al., 1990) and at the perforant pathway innervation of the DG (Burgard and Sarvey, 1990)

4. Reduction of principal cell adaptation in DG, CA3 and CA1 (Madison and Nicoll, 1984; Madison et al., 1987)

5. ACh suppresses inhibition of dentate granule cells (Bilkey and Goddard, 1985) and also decreases perisomatic inhibition of pyramidal cells, probably by suppressing release from basket cell terminals (Behrends and ten Bruggencate, 1993). Accordingly, in the model ACh suppresses inhibition in all model layers.

These combined effects may put the network in a state that promotes new learning; the suppression of transmission in intrinsic fibers by ACh could make the hippocampus more "attentive" to online input, while its effects on LTP could enhance learning. Its effects on adaptation and inhibition, as well as the depolarizing effects, enable hippocampal principal cells to fire at greater frequency at lower input levels. Thus, high ACh may set the hippocampal network to a learning mode (Fig. 2A), while low ACh could set the appropriate dynamics for recall (Fig. 2B). (For more detailed discussion of various ACh effects, see Hasselmo and Bower, 1993; Hasselmo, 1995).

\section{How Does the Model Store and Retrieve Patterns?}

One aim of the model is to explain how correct dynamics for learning are set as a function of novelty detection. A primary assumption herein is that if the organism is in a known environment, and is not engaged in a new task, the hippocampus will be in what has been called retrieval mode. In this mode, patterns in EC representing the outer and inner milieu lead to retrieval of associated patterns in the hippocampus, enabling the organism to anticipate unobserved, but known, features of its surroundings. The hippocampal excitatory activity accompanying the reinstatement of stored patterns induces matched activity in the inhibitory nodes. This inhibition not only limits hippocampal activity, but also depresses the septal cholinergic node. Maintained low ACh levels mean that little learning takes place.

If the animal is suddenly faced with an unknown situation, the observation-and-retrieval stream breaks down, because there are no stored patterns in the hippocampus that match the on-line EC pattern. Because of decreased activity in CA3 and CA1 the septum is disinhibited, resulting in elevated $\mathrm{ACh}$ release in the hippocampus, and therewith in a shift to learning mode.

In learning mode, the effects of ACh outlined above collectively facilitate storage of the new pattern. Subthreshold depolarization, combined with suppression of feedback inhibition and adaptation, induces some nodes in DG and the hippocampal fields to fire in response to the new EC pattern. Through Hebbian learning, enhanced by $\mathrm{ACh}$, connections between the patterns in all layers are strengthened, leading to storage of a representation of the new pattern. Restored firing in CA3 and CA1 then begins to inhibit the septum again, leading to a decrease in $\mathrm{ACh}$ release and a gradual return to retrieval mode.

An unresolved issue is how long the hippocampus takes to store a pattern. This is not a critical variable in the model, as there is a trade-off between time available for storage and the learning rate, another unknown. In the present simulations, the maximal learning rate of the model, i.e., with maximal ACh modulation, allows storage of an input pattern over a single theta cycle, while with intermediate $\mathrm{ACh}$ modulation several theta cycles are necessary for reliable storage. These settings are loosely based on in vivo LTP induction with stimuli patterned after natural activity (brief burst stimulation on the positive phase of theta). Such stimuli induce LTP within one theta cycle (Huerta and Lisman, 1995), while LTP is saturated after approximately 3 cycles (Holscher et al., 1997).

\section{RESULTS}

To investigate the model outlined above, several simulations were run. The first two center on behavior of the model in the learning and retrieval modes. Subsequent simulations evaluate novelty detection, the effect of novelty on ACh release, the effects of ACh on activity and learning, and the switch from one mode to another. For each simulation, results were averaged over at least twelve runs, each with random activation values at onset. All simulations were performed with the same parameter settings.

\section{Storing and Retrieving a Pattern}

In the first simulation, we evaluate what happens, over one theta cycle, in the various hippocampal layers of the model when a pattern is stored or retrieved. Notably, ACh levels in the model do not change substantially on this time scale. The pattern was activated by clamping a single input node, representing neocortical input sources, that was connected to a random subset of EC nodes via uniform weights. The input node was switched on in the down 
A

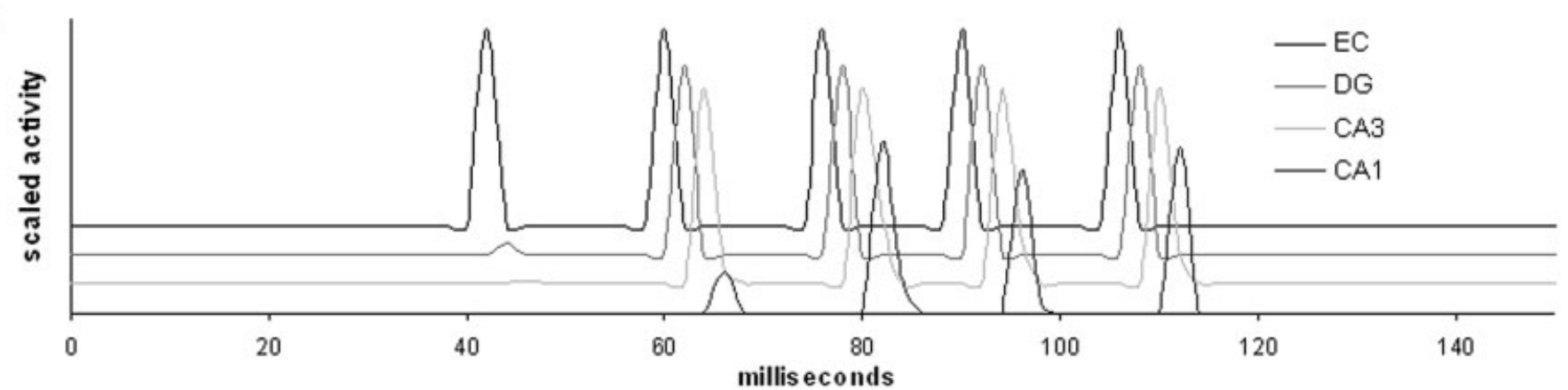

B

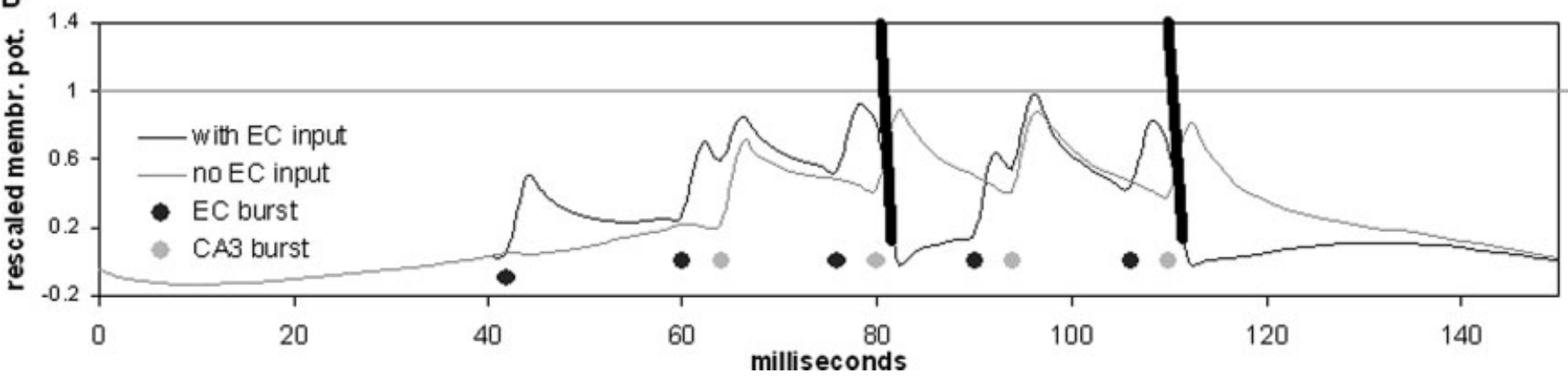

FIGURE 3. Storage of a new pattern. A: Activity in all model layers during the simulated theta cycle. At iteration 0 , the pattern is presented. This leads, during the up-phase of the theta cycle, to gammafrequency firing in entorhinal cortex (EC), followed after one gamma cycle by firing in dentate gyrus (DG) and CA3. Activity of CA1 takes longer to build up. After several gamma frequency activity bursts, the system shuts down due to mounting inhibition. B: Membrane potential

phase of the theta cycle, where it could not lead to immediate firing in EC. In this simulation, the model started out in learning mode, with ACh modulation in the hippocampus set to a value of 0.75 .

Following learning over one theta cycle, the pattern was presented again in retrieval mode, with the ACh concentration set to 0.1 at the start of the trial. This simulated a later attempt at pattern retrieval. A second, random pattern was also presented in retrieval mode (ACh set at 0.1 ), to control for the possibility that patterns not learned would also be "recalled," or that the stored pattern would be recalled with a random cue.

\section{Storage}

The activity level of the different layers during the learning trial is shown in Figure 3A. At the start of the trial, in the down phase of theta, all layers are silent. During the rising phase of septal theta, hippocampal inhibition decreases. As can be observed in Figure $3 \mathrm{~A}$, the constant input, in interaction with both local feedback inhibition and cell adaptation, then leads to gamma-range firing bursts in the EC (Dickson et al., 2000; Whittington et al., 2001). DG nodes respond to the input from EC with one gamma cycle delay. Within the same gamma cycle, activity of the dentate triggers the formation of firing patterns in CA3 and some initial activity in CA1, which increases over the subsequent cycle. Toward the end of the simulation activity in the various layers subsides under the influence of increasing theta inhibition. of two CA1 nodes during acquisition: one receives an innervation from an EC pattern node (black), one does not (gray). The timing of population bursts in EC and CA3, the inputs of the CA1 layer, are depicted as dots, in black and gray respectively, along the time axis. Only the membrane potential of the innervated node crosses firing threshold (horizontal gray line, with think black lines marking emissions of a spike).

\section{CA1 binds hippocampal patterns to cortical representations}

The patterns in DG and in CA3 are formed by self-organization. This means that random weights in the feedforward connection determine which nodes are part of the pattern, without requiring any outside supervisor. These self-organized patterns need to be associated with the cortical representations that give rise to them, to enable pattern completion in the cortex (McClelland and Goddard, 1996). As explained earlier, this may occur through the reciprocal, topologically organized, connections between CA1 and the EC. Therefore, we used the number of firing CA1 nodes that receive a one-to-one connection from an entorhinal pattern node as read-out measure, both to monitor learning and to evaluate retrieval. These nodes will be referred to as "correct CA1 nodes," whereas other firing CA1 nodes will be referred to as "incorrect CA1 nodes." As there are $12 \mathrm{EC}$ nodes per pattern, the number of correct CA1 nodes varies between 0 and 12 .

Figure 4 shows that most nodes firing in CA1 during retrieval indeed receive a connection from the EC. This is not a direct result of triggering by entorhinal input, however. In panel b of Figure 3, the membrane potentials of two CA1 nodes are plotted over time, during acquisition of an input pattern. One of the nodes receives entorhinal excitatory input, while the other one does not. The timing of both $\mathrm{EC}$ and $\mathrm{CA} 3$ bursts has been marked. While the EC 


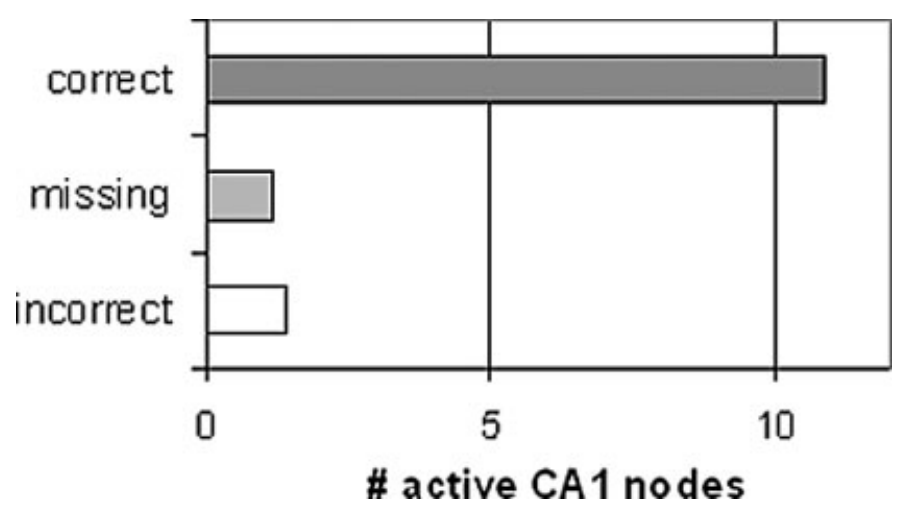

FIGURE 4. Number of active nodes in CA1 during learning, split out in the 12 CA1 nodes that are innervated by entorhinal cortex (EC) pattern nodes ("correct"), and those that are not ("incorrect"). "Missing" refers to those CA1 nodes that did not fire during learning, even though they are innervated by one of the EC pattern nodes.

bursts cause subthreshold depolarization in the CA1 node receiving a connection from EC, the node only crosses the firing threshold after receiving additional input from CA3. In the other CA1 node, EC input causes hyperpolarization due to the broad feedforward inhibition accompanying targeted excitation in perforant path projections.

Notably, not all CA1 nodes receiving excitation from EC become active. This is the result of sampling variance: though most
CA1 nodes receive sufficient excitation from CA3 to cross threshold, some are not innervated by a sufficient number of active CA3 nodes. Strengthening the Schaffer collaterals would make the latter nodes reach threshold, but would lead to many nodes not targeted by the EC pattern to fire as well. This mechanism for binding hippocampal patterns to cortical representations thus crucially depends on the balance in strength of the two inputs to CA1.

\section{Retrieval}

Figure 5 shows the activation in the network after presentation of the old and the new pattern in retrieval mode $(\mathrm{ACh}=0.1$ ). When the old pattern is presented, DG, CA3, and CA1 all respond to EC activity within one gamma cycle (Fig. 5A). In contrast, the new pattern elicits little or no activity (Fig. 5B). Activity levels in the hippocampus thus distinguish between old and novel input patterns. This provides the novelty signal underlying the mode-shifting mechanism, which comes down to a drop in firing of hippocampal pyramidal cells when novel patterns are presented.

The differential firing to old and novel patterns is already present in DG, suggesting that this structure plays a major role in novelty detection. This follows in a rather straightforward manner from anatomical and physiological considerations: The broadly fanning and modifiable perforant path connections allow for a strong difference between the amount of signal produced by old and novel patterns. Later steps in the three-synaptic pathway are

\section{A Old pattern}
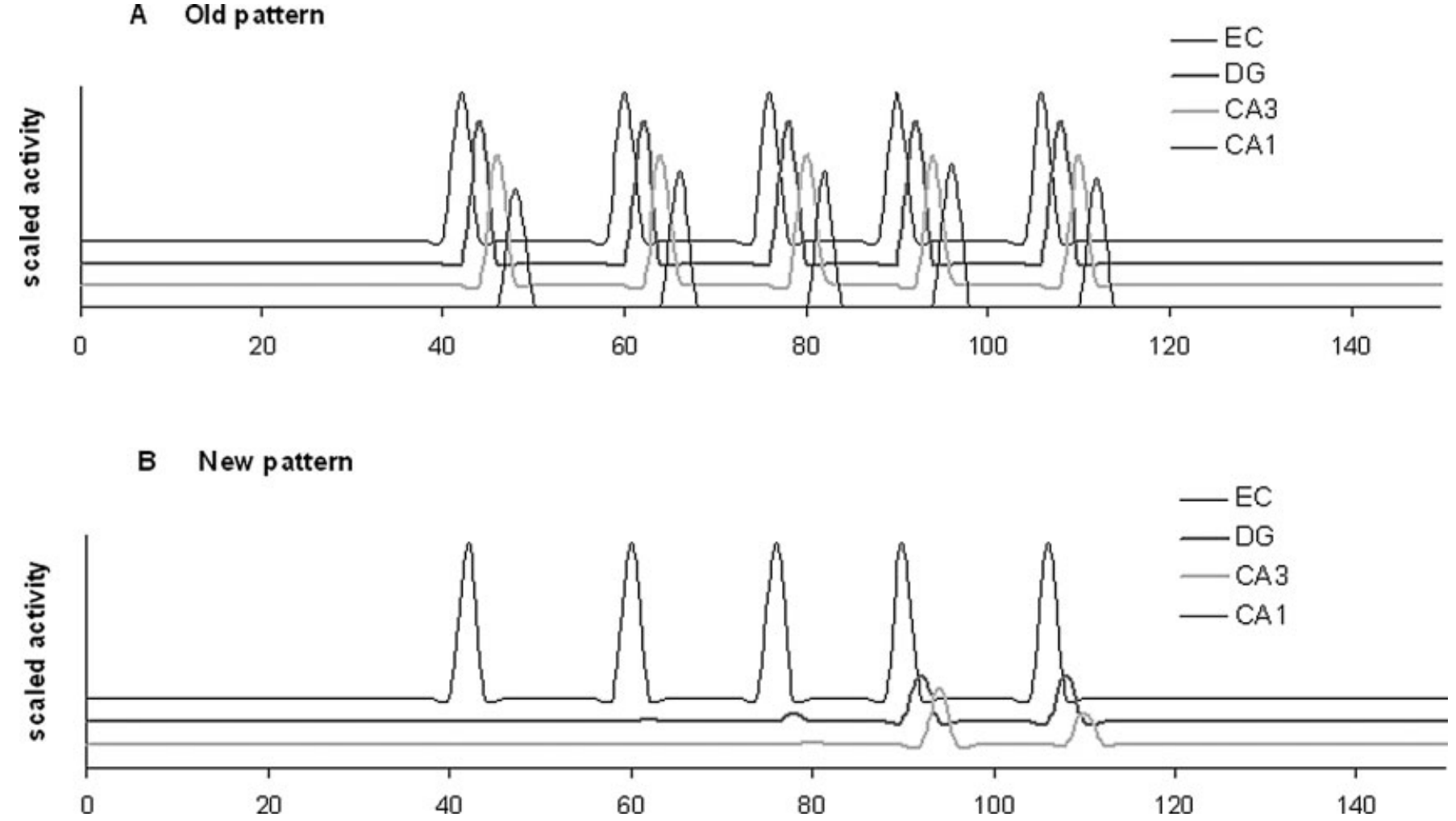

FIGURE 5. Activity in retrieval mode in all model layers during two simulated theta cycles. A: In the first theta cycle, a stored pattern is presented, which induces notable activity in all model layers. B: In the second theta cycle, a new pattern is presented. Now entorhinal cortex (EC) activity triggers little or no activity in the other modules. 
less suited for novelty detection, as the point-to-point organization and lack of Hebbian plasticity in the DG to CA3 pathway do not support the specific association of dentate and CA3 patterns. Moreover, the strong DG-to-CA3 connections might induce a fast response in CA3 even before (non-Hebbian) learning has occurred; one not related to reactivation of old patterns. The monosynaptic pathways from EC to the Ammon's horn (CA3 and CA1) are also unsuitable for novelty detection, because hippocampal pyramidal cells are not usually triggered by EC input alone (Canning et al., 2000).

\section{Pattern Completion}

A second simulation analyzes the effects of hippocampal mode on pattern completion. After acquisition of one pattern, we deactivated a variable proportion of the EC nodes associated with that pattern and tested retrieval for each level of degradation. Retrieval was tested over one theta cycle, with the ACh level set to either 0.1 (retrieval mode) or 0.75 (learning mode). Pattern completion was measured as the maximum proportion of correct CA1 nodes (receiving an input from the complete EC pattern) that were simultaneously active during the theta cycle.

Figure 6A depicts pattern completion in retrieval mode as a function of cue size; that is, the number of EC pattern nodes that were allowed to become active. As can be observed, there is a nonlinear relation between cue size and pattern completion. Near full pattern completion is achieved with cue sizes of $60-100 \%$. There is a steep decline of function with cue sizes around $50 \%$, while with still smaller cues pattern completion is almost zero. In the simulation CA1 activity is strongly correlated with elicited activity in DG, which responds to the degree of input degradation with a similar nonlinear function (gray line in Fig. 6A). A nonlinear relation between cue size and pattern completion was previously shown to result from the interaction of orthogonalization in DG with the workings of LTP and LTD (O'Reilly and McClelland, 1994), which are both implemented in the current model. It thus appears that most pattern completion occurs already in DG.

Figure $6 \mathrm{~B}$ shows what happens to pattern completion when the model is in learning mode. The high ACh modulation enhances activity levels in the model so that partial pattern completion tends to occur with relatively smaller cue size than in retrieval mode. In essence, dampening of transmission by $\mathrm{ACh}$, which makes activation of pattern nodes harder, is trumped by the depolarizing effects of ACh, which makes such activation easier. However, firing is also made easier for nodes outside of the already stored patterns. Consequently, retrieval becomes less accurate, as reflected in a considerable increase of incorrect nodes being activated during pattern completion (Fig. 6B). Thus, with small cue-sizes more of the pattern is completed in learning mode than in retrieval mode, but this comes at the price of a compromised integrity of retrieval.

It may be hypothesized that during effortful retrieval, input cues do not immediately lead to instatement of a stored pattern, leading to a shift of the system to learning mode, with higher ACh. This would be consistent with the "retrieval practice effect" known in the human memory literature, which implies that effortful and successful retrieval constitutes a powerful learning method

\section{A retrieval mode}

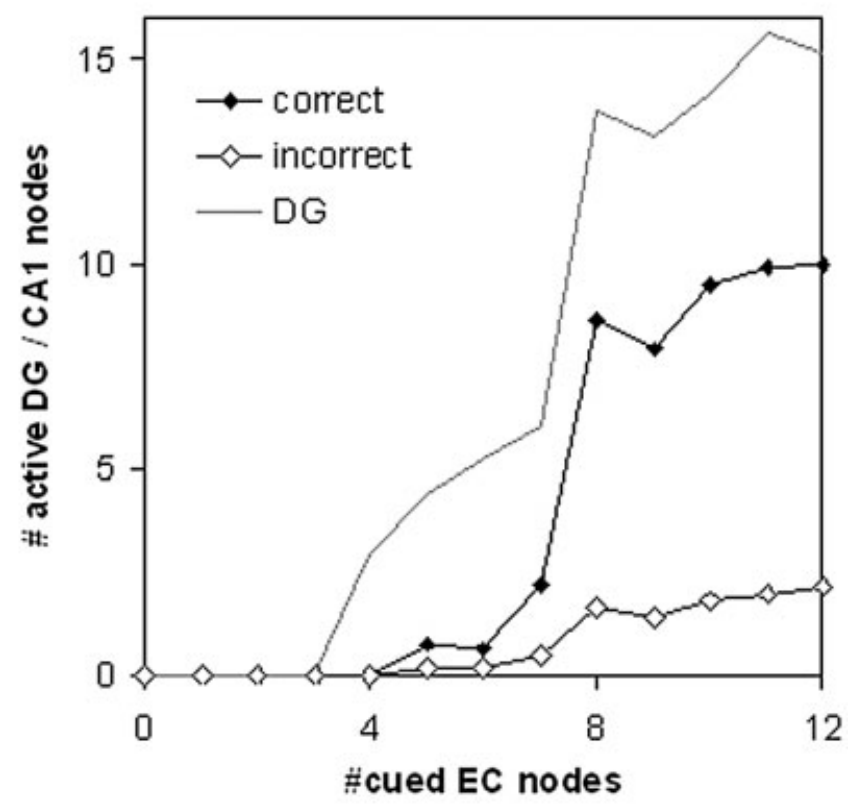

B learning mode

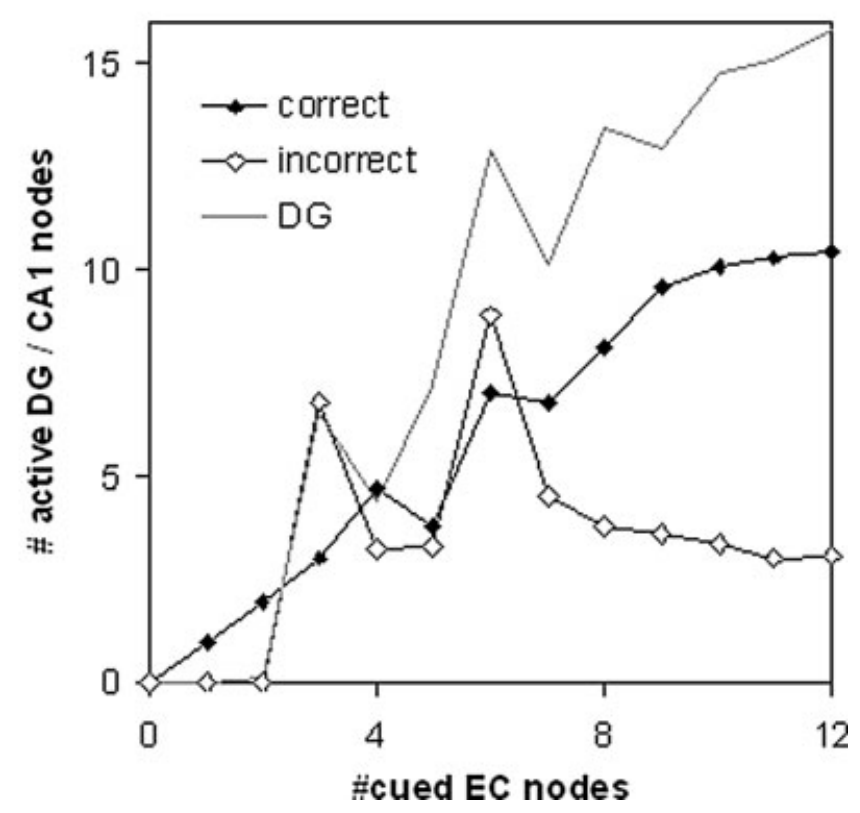

FIGURE 6. Activity elicited in dentate gyrus (DG) and CA1 by a partial cue in entorhinal cortex (EC), activated during retrieval mode (A), or learning mode (B). The $\mathrm{x}$-axis marks the number of $\mathrm{EC}$ nodes in the stored pattern that were activated as a cue. The $y$-axis marks the maximal number of simultaneously active DG and CA1 nodes during the simulated theta cycle. "Correct" refers to those nodes innervated by an EC pattern node (independent of whether this node was cued or not), while "incorrect" refers to other CA1 nodes. 
(Gardiner et al., 1973). That is, effortful retrieval has a stronger effect on retention than fast, easy recall (Benjamin et al., 1998).

\section{Novelty Detection and Dentate Gyrus}

Novelty detection in the dentate was investigated systematically by tracking the model's response to inputs that are a mixture between old and new patterns. After acquisition of one pattern, as in the previous simulation, we cued the network with patterns that were either completely old (i.e., equal to the pattern stored earlier in the simulation), or completely new, or that contained a variable ratio of old and novel EC nodes. Again, ACh during retrieval was set to either 0.1 (retrieval mode) or 0.75 (learning mode). For each of the input patterns, the maximal number of active nodes over the first theta cycle was registered in the dentate, as well as in CA1 (activity in CA3 strongly correlates with activity in CA1, and was therefore not plotted). For DG, we considered nodes related to the old pattern separately from other nodes. All DG nodes that had been active during acquisition were taken to reflect the old pattern.

In retrieval mode (Fig. 7A), there is a steeply sigmoidal relation between the degree of input novelty and total DG activity, which is largely determined by the response of nodes related to the old pattern (strong activation of old DG nodes for predominantly old input). Some activity is also apparent for predominantly novel patterns (left side of Fig. 7A), but it is unrelated to the old pattern: there is very little overlap of the areas under the curves for "same" and "different" nodes. Figure 7A also shows that activity in CA1 strongly correlates with activity of "old" DG nodes only: no CA1 activity is elicited by relatively novel patterns. The few DG nodes that fire to novel inputs trigger some CA3 nodes (Fig. 5B), but these are not sufficient to elicit firing in the CA1 layer. Thus, only stored DG activity propagates through the circuitry, retrieving the original representation through pattern completion. On an abstract level, this reflects a nearly binary decision as to whether a pattern is old or new: predominantly old EC patterns lead to retrieval of old DG nodes, and subsequently strong activity in CA3 and CA1; predominantly new EC patterns to activation of solely new DG nodes, and little or no activity in the fields of the hippocampus proper. Only a narrow range of mixed inputs elicits possibly ambiguous system activity.

The behavior of the network is again very different in learning mode $(\mathrm{ACh}=0.75)$. As shown in Figure $7 \mathrm{~B}$, all types of input patterns elicit strong activity in DG and in CA1. Thus, there is a novelty signal (a sharp difference in the activity elicited by novel and by old patterns) only in retrieval mode. Furthermore, the response curves to the degree of input novelty of both "same" and "different" DG nodes are more linear than in retrieval mode. The combined effects result in a larger overlap of the areas under the curves for same and different nodes. In other words, a larger range of mixed input patterns activates a mixture of old and new DG nodes.

\section{Effects of ACh on Pattern Storage}

In one simulation, we systematically assessed the effect of ACh modulation on pattern storage. A new pattern was presented during one theta cycle (the acquisition theta cycle), with ACh modu-

\section{A retrieval mode}

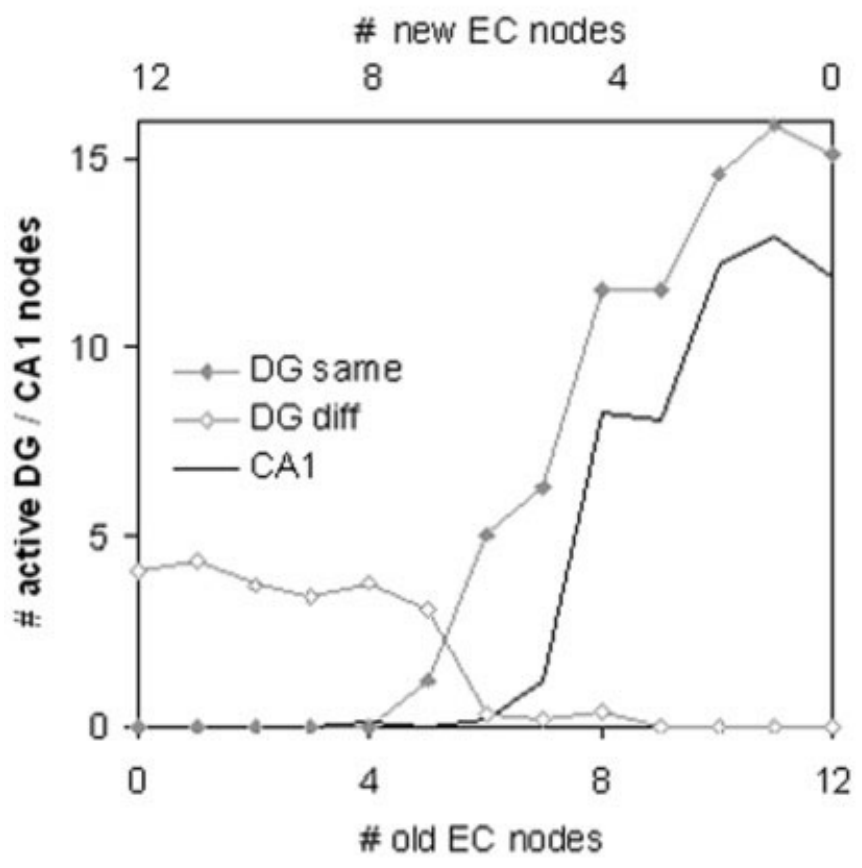

B learning mode

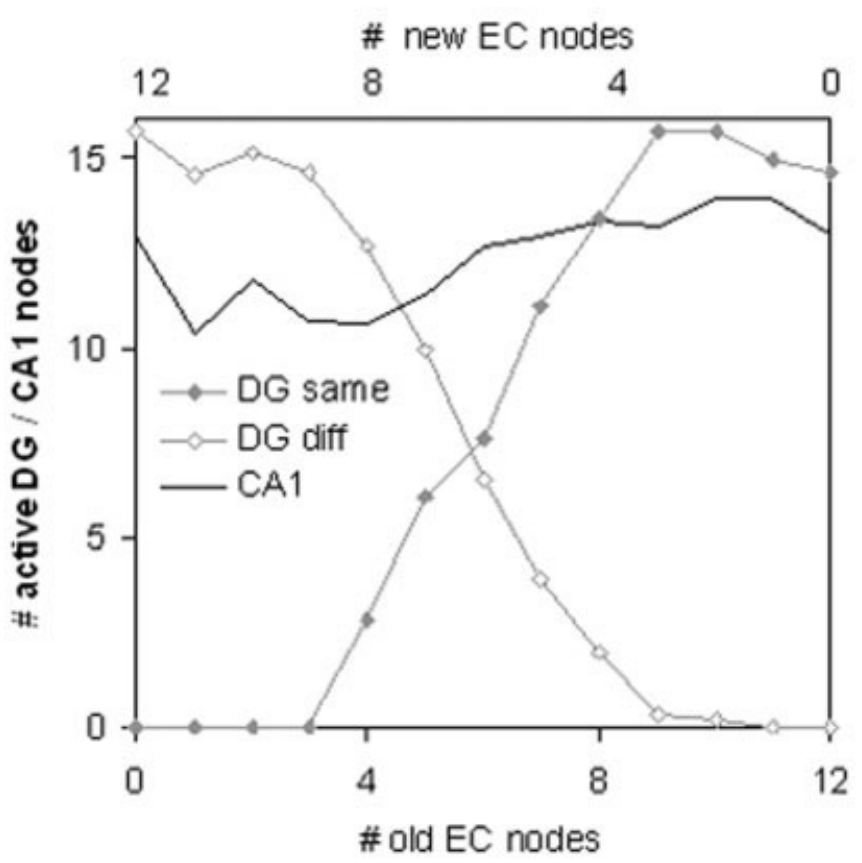

FIGURE 7. Activity elicited in dentate gyrus (DG) and CA1 by patterns consisting of $n$ old entorhinal cortex (EC) nodes and 12-n novel EC nodes, activated during retrieval mode (A), or learning mode (B). The $x$-axis marks the number of old (lower scale) and new (higher scale) nodes in the presented pattern. The $y$-axis marks the maximal number of simultaneously active DG and CA1 nodes during the simulated theta cycle. "DG-same" refers to DG nodes also active during acquisition of the stored pattern, while "DG-diff" refers to other DG nodes. 


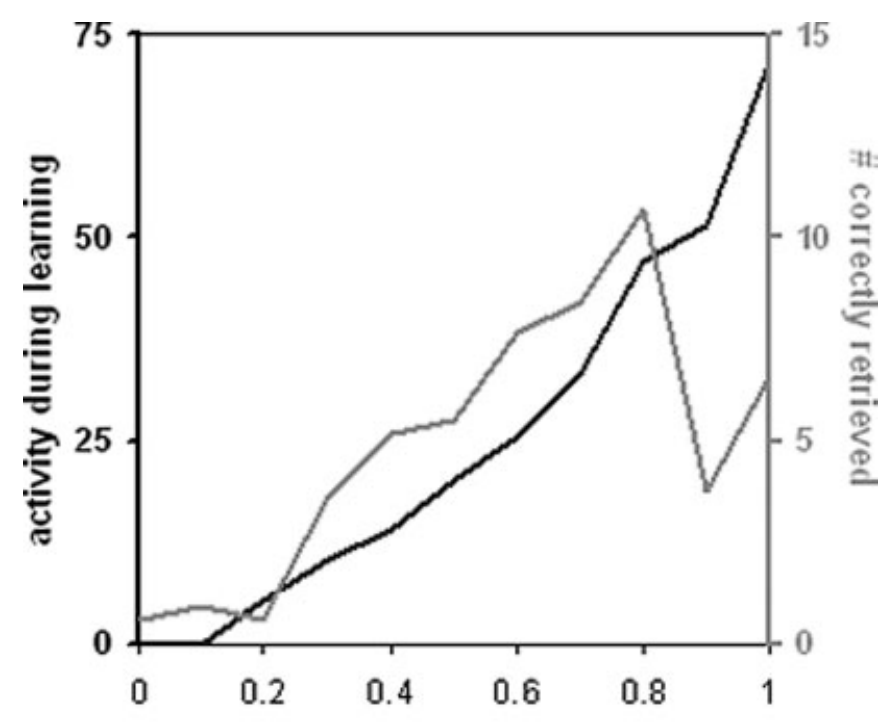

ACh modulation during le arning

FIGURE 8. Effects of acetylcholine (ACh) modulation on learning. A new pattern was presented during one theta cycle, at various levels of ACh modulation. Activity in CA1 summed over the theta cycle, and the number of activated correct CA1 nodes during a subsequent retrieval theta cycle (with ACh modulation set to 0.1 ), are plotted against ACh modulation.

lation set at different levels at the onset of simulation (little change to modulation occurred over one theta cycle). For each level of ACh modulation, acquisition was evaluated by presenting the pattern again during one theta cycle (the retrieval theta cycle), with ACh modulation set at 0.1 . Two measures were extracted from the simulation data: (1) the total number of active nodes in CA1, summated over the duration of the acquisition theta cycle; (2) the maximum number of correct CA1 nodes simultaneously active during the retrieval phase. This second measure assessed ACh effects on learning performance.

As shown in Figure 8, higher ACh modulation results in higher CA1 activity during learning. This occurs through the combined effects of increased depolarization, decreased adaptation of excitatory nodes, and decreased inhibition. These higher activity levels, combined with the higher learning rate at high ACh modulation levels, lead to larger representations being formed and to enhanced retrieval performance. Thus, hippocampal learning is positively correlated with ACh level.

The right-hand side of Figure 8 shows an interesting exception, however. The highest ACh levels tested result in suboptimal retrieval performance, giving rise to an inverted U-shaped curve. In fact, at the highest ACh levels CA1 nodes are depolarized by ACh to the extent that they can be triggered by EC input alone. As some CA1 nodes thus fire before the arrival of input from the CA3 layer, Hebbian learning in the Schaffer collaterals connecting to these nodes cannot occur. By the time CA3 activation does arrive, the CA1 nodes that fired "prematurely" are less likely to fire because of adaptation (which eventually sets in, despite the dampening influence of $\mathrm{ACh}$ ). Neurons that only get input from CA3 are now relatively more likely to fire and to form strengthened connections with CA3. These neurons do not represent the EC input, however, leading to diminished retrieval performance. ACh levels higher than 0.85 do not naturally occur in the model and, thus, neither does the described "pathological" network behavior. Nonetheless, this mechanism might underlie the inverted U-shaped dose-response relation typically observed for cholinomimetic agents in memory tasks (Wanibuchi et al., 1994).

\section{Effects of ACh on Novelty Detection}

To investigate the interaction between $\mathrm{ACh}$ and the novelty signal, we stored one pattern (in learning mode; $\mathrm{ACh}=0.75$ ), and then presented either this "old" pattern, or a randomly selected new pattern, while systematically varying the ACh modulation at simulation onset. Each pattern was activated at the trough of theta. During the theta cycle that followed, activity in the various hippocampal modules and ACh release were monitored.

Figure 9A shows activity levels in the hippocampal modules during presentation of either the new or the stored patterns, plotted against initial ACh modulation. Activity levels in CA1 were summated over the duration of the test (one theta cycle). For retrieval of the stored pattern, ACh modulation is not very important: the pattern elicits activity in the network both in learning mode (high ACh) and in retrieval mode (low ACh). This is different for the new pattern, which triggers firing in DG and CA1 only in learning mode. Again, the difference in activity elicited by old and new patterns is first apparent in the DG. It is activity in this module that differentiates between old and new patterns, but only in situations of low ACh.

ACh release from the septum during the theta cycle mirrors the activity elicited in the hippocampus by the input patterns (Fig. 9B): it is low when there is activity in CA3 and CA1 (due to the inhibition of septal activity by these layers), but it is high when there is no activity in CA3 and CA1 (i.e., when a new pattern is presented in retrieval mode). This last situation is when a mode shift is necessary, and the release of ACh will cause just that.

\section{Time Course of Mode Shifting}

From the previous simulation, the principle underlying mode switching becomes clear. If in retrieval mode no activity is elicited in Ammon's horn, inhibition of the septum is released, $\mathrm{ACh}$ is released, and the network will shift to learning mode. Conversely, the shift from learning mode to retrieval mode occurs when activity in the hippocampus increases, for instance due to buildup of a representation of novel input. The increasing hippocampal activity progressively inhibits ACh release, in time bringing the system back to retrieval mode.

In the present study, we simulated this process explicitly. At the onset of the simulation, ACh modulation was set to a low value (0.1) to simulate retrieval mode. Then a new pattern was presented to the model, at the trough of theta, and was kept active during 20 consecutive theta cycles $(4 \mathrm{~s})$. This procedure provides an opportunity to evaluate the temporal characteristics of mode shifting. The procedure was repeated with presentation of an old pattern, as a control condition. 


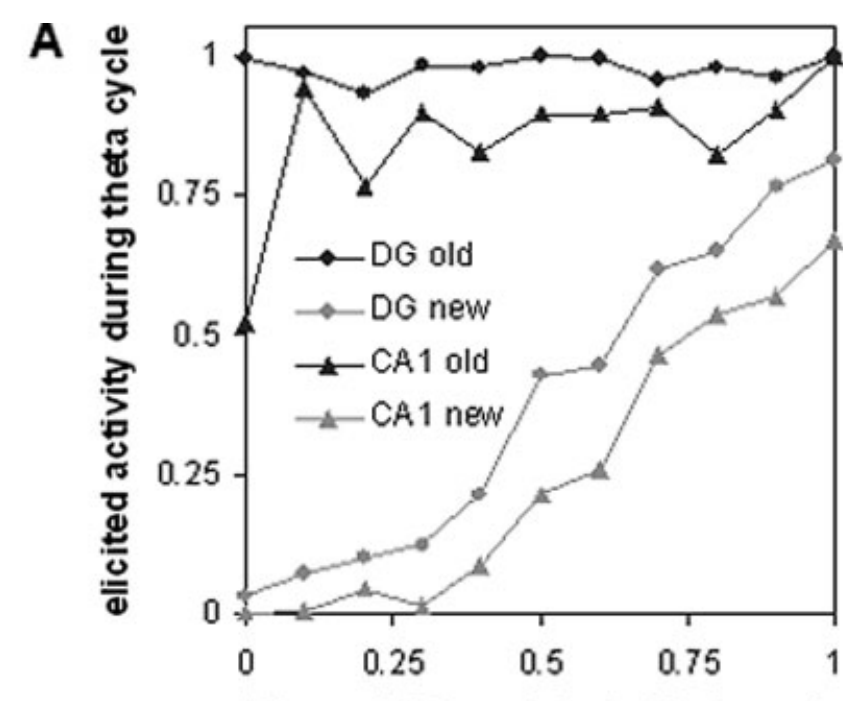

ACh modulation at start of theta cycle

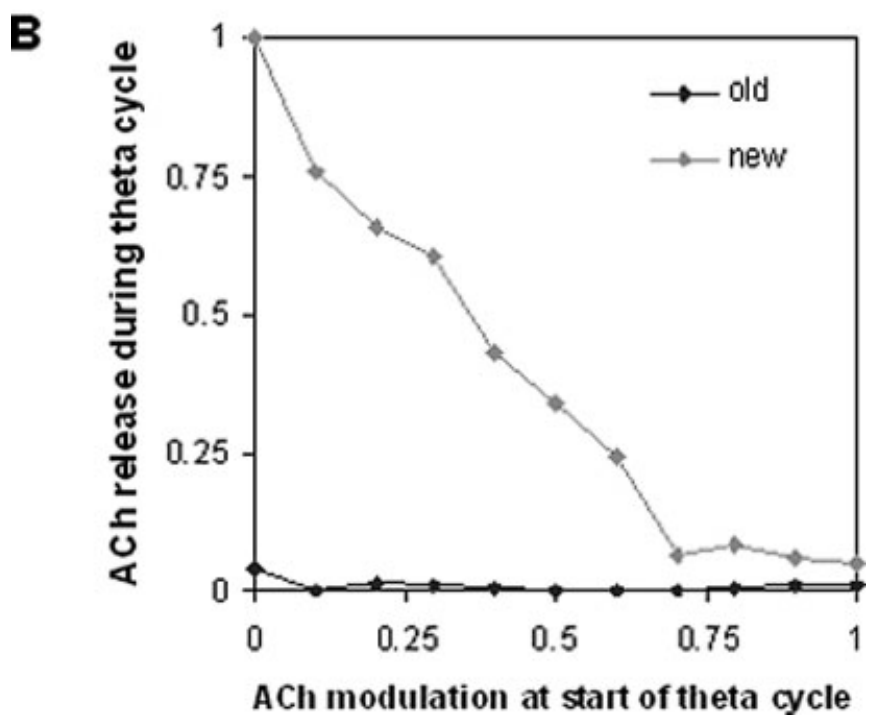

FIGURE 9. Effects of different levels of acetylcholine (ACh) modulation on model behavior. A: Activity elicited in dentate gyrus (DG) and in CA1, following presentation of either an old (black lines) or a new (gray lines) pattern at varying initial ACh levels. Activities in DG and in CA1 were summated over one theta cycle following pattern presentation. B: ACh release in the hippocampus during the same theta cycle for the old (black line) and the new patterns (gray line). Variables were normalized, dividing them by their maximum value, in all simulations.

Figure 10C presents activity in the model layers during the course of simulation. Activity was now summated over layers CA3 and CA1. In the control condition, in which an old pattern was presented, activity remained high (not shown), while septal cholinergic activity (gray line in Fig. 10A) and hippocampal ACh modulation (gray line in Fig. 10B) remained subdued throughout the whole simulation.
Results were different for presentation of the new pattern. As shown also in the first simulation, the new pattern does not initially elicit activity in DG and Ammon's horn. This lack of activity in Ammon's horn leads to a drop in inhibition of the septum, which in turn leads to a spike in activity of the septal cholinergic node (Fig. 10A). The increase in septal activity results in a gradual rise of ACh modulation in the hippocampal layers (Fig. 10B). As was detailed in previous simulations, rising ACh modulation enables the formation of a new pattern in the hippocampal layers, leading to a rise in their activity at around $800 \mathrm{~ms}$. The return of activity in the layers of Ammon's horn leads to renewed inhibition of the septum and a drop in septal activity and ACh release. ACh modulation remains elevated for a few seconds more, due to its slow synaptic and postsynaptic dynamics. Eventually, it starts to drop under the influence of continued inhibition of the septum by the hippocampal layers (maximum modulation was reached at 3,960 $\mathrm{ms})$. Assuming negligible $\mathrm{ACh}$ release to continue after the modeled episode, ACh modulation returns to a value of 0.2 at $20 \mathrm{~s}$ after simulation onset, resetting the model to retrieval dynamics.

Although activity in the hippocampal layers continues to rise over the entire modeled interval of $4 \mathrm{~s}$, the time required to store a representation of the new pattern is much briefer than this. Figure $10 \mathrm{D}$ shows how many "correct" CA1 nodes were recruited at each theta cycle. The largest increase takes place from 1000 to $1600 \mathrm{~ms}$, when most of the representation is formed. When we set the model to retrieval mode after $1800 \mathrm{~ms}$ and test for retrieval of the pattern, the number of correctly activated CA1 nodes has already reached $84 \%$ of its maximal value. The simulation demonstrates how a change from retrieval to learning mode occurs within $2-4 \mathrm{~s}$, while it takes relatively more time to return from learning to retrieval mode.

\section{DISCUSSION}

Novelty detection and mode shifting were studied in a largescale connectionist model of the hippocampal formation. The results show how old and novel patterns may be distinguished based on differential activity levels in the system, and how this differential signal may be used to regulate levels of a neuromodulator, which, in turn, acts to alter system dynamics of the hippocampalentorhinal circuitry. The simulations show a relatively slow time course of mode shifting (seconds, to tens of seconds), whereby mode shifts occur on the same time scale as natural episodic learning.

According to the present model, input patterns that have been previously learned induce higher levels of activity than novel ones in the first few theta cycles after presentation. The reason is that, only for old patterns, strengthened connections exist from the participating EC neurons to particular DG granule cells. The EC input to CA3 is less important in this respect, because the CA3 nodes are dependent on input from the DG to reach firing threshold. This implicates that the "novel versus old" decision depends crucially on the dentate and on rules governing EC to DG synaptic plasticity. In line with this notion, a number of experimental studies suggest that dentate granule cells may, indeed, play a significant 
A

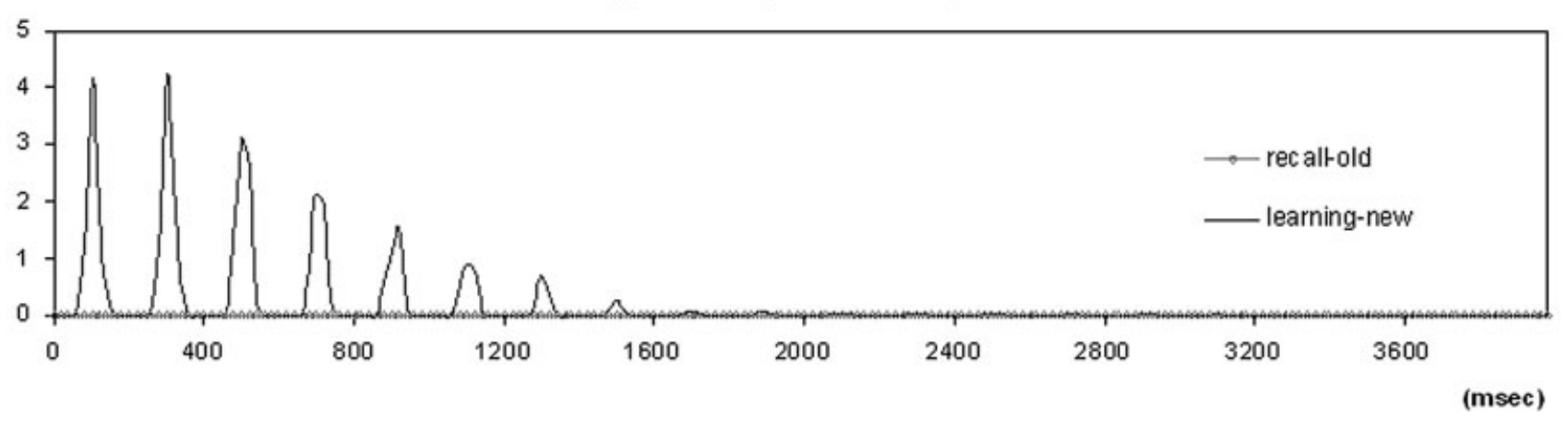

B ACh modulation in the hippocampus

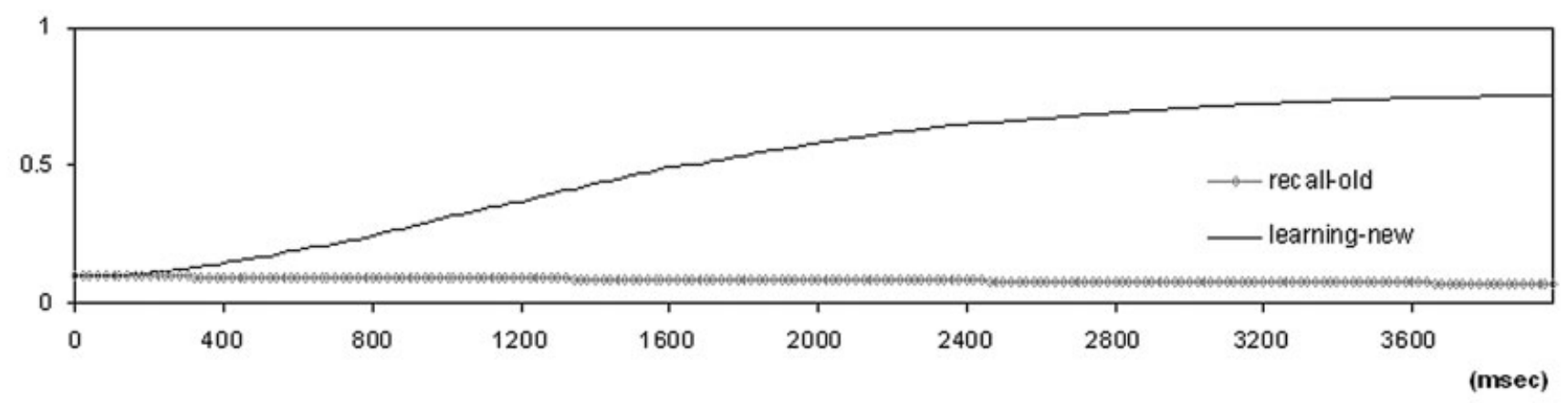

C

Activity in model layers

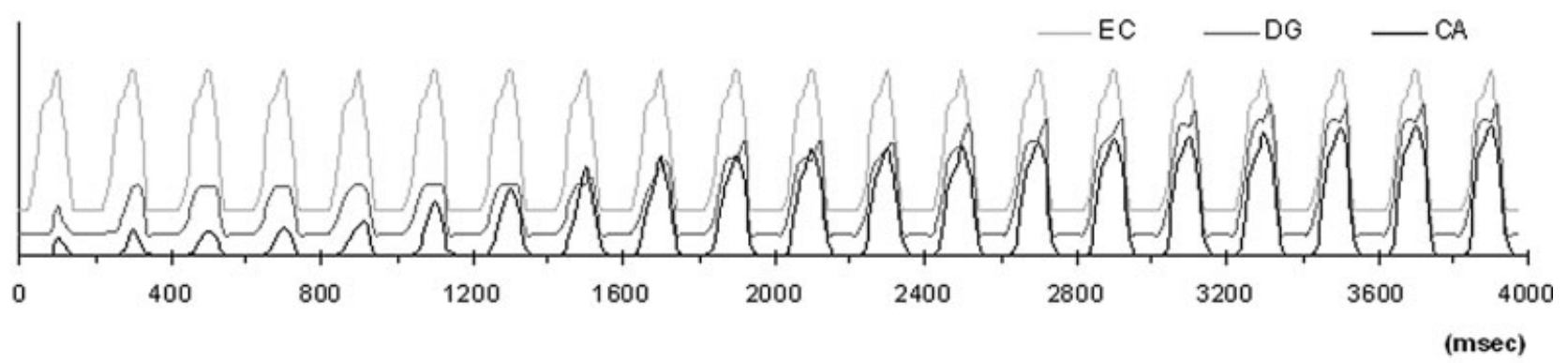

D Recruited correct CA1 nodes

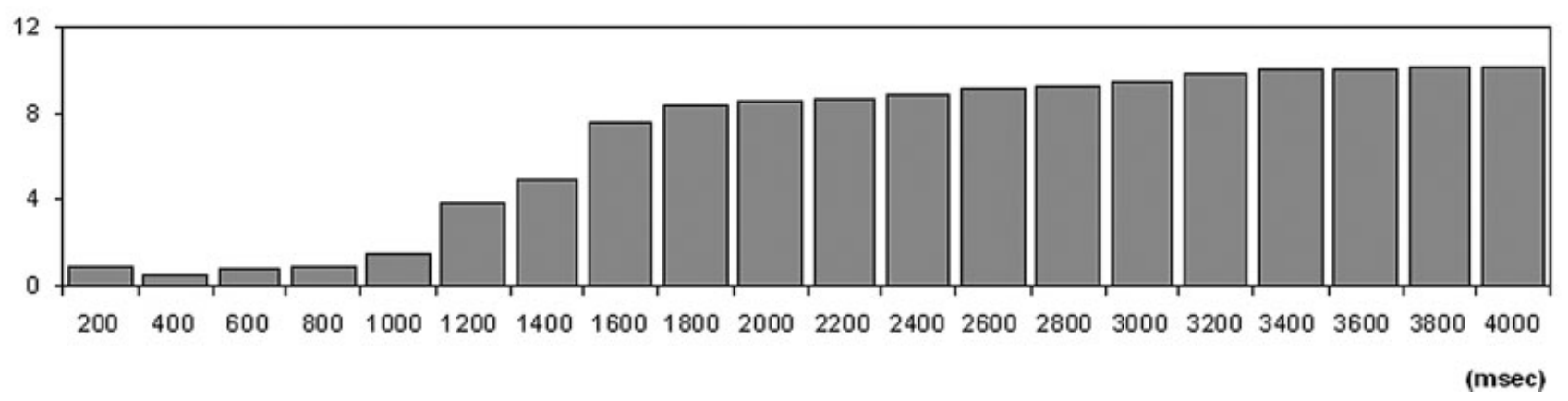

FIGURE 10. Example of a shift from retrieval mode to learning mode. At time $=0$ either a new or an old pattern is presented to the model. Data are averaged over 20-ms intervals. A: Activity of the septal node in response to either an old (gray) or a new pattern (black). B: Acetylcholine (ACh) modulation in the hippocampus during presentation of an old (gray) or new (black) pattern. C: Normal- ized activity in entorhinal cortex (EC), dentate gyrus (DG), and the two CA layers (summation of CA3 and CA1) during presentation of a new pattern. D: Maximum number of active correct CA1 nodes at each subsequent theta cycle (100 iterations within one theta cycle), during the course of simulations ( 20 theta cycles). 
role in novelty detection (Johnson and Moberg, 1980; Lemaire et al., 1999). Notably, the orthogonalization between EC and dentate tends to drive the overlap between dentate representations toward all or nothing in retrieval mode. Hence, the novelty detection procedure tends to produce an almost binary "old-versusnovel" decision.

Not the dentate, but the CA3 and CA1 regions appear to be the largest sources of hippocampal feedback to the medial septal nucleus (Alonso and Kohler, 1982; Tóth and Freund, 1992; Tóth et al., 1993). These subfields may contribute to novelty detection, in vivo, by increasing the amplitude of the novelty signal. Both the denser activity with respect to the dentate and the propensity of pyramidal cells to fire in bursts may contribute to this amplification.

Besides pointing out the prominent role of DG in novelty detection, the present work also provides a new reasoning for the existence of separate learning and retrieval modes. While other studies have focused on the detrimental effect of retrieval during learning (e.g., Hasselmo and Schnell, 1994) and on unwanted changes in existing representations (e.g., Grossberg, 1976), the current work shows how retrieval in learning mode may be unreliable through the activation of features that were not part of the original memory. The separation of learning and retrieval modes, thus, also enhances accurate retrieval.

Other novel insights follow from the incorporation of oscillatory population dynamics in the model. In particular, to ensure that ACh modulation fluctuates in relation to novelty, but not with faster theta or gamma rhythms, the hippocampo-septo-hippocampal loop needs to incorporate a low band filter. In the model this is implemented in the form of slow inhibition in the pathway controlling ACh release, and slow time constants governing ACh effects. In the biological circuitry, the first procedure may, for instance, consist in a long integration period for inhibitory hippocampal input to ACh cells, while the slow effects of ACh were shown to result, at least in part, from slowly recovering intracellular processes, activated by ACh in the postsynaptic cell (Hasselmo and Fehlau, 2001). Phase shifts in the theta oscillation between different hippocampal regions might also contribute to smoothly fluctuating ACh levels by "widening" the phase of theta during which septal cells receive inhibition from the hippocampus.

The aspect that most clearly distinguishes the current model from others incorporating learning and retrieval modes, is the slow time course of the shift between modes. A previously published model takes an opposing standpoint, emphasizing the necessity for fast functional mode shifts (Hasselmo et al., 2002). In the latter model, a shift from learning to retrieval mode occurs within each theta cycle (i.e., every $100-300 \mathrm{~ms}$ ), while in our own model it takes multiple seconds to occur. The other main differences between this "fast shift model" and our own are as follows. First, ACh does not play a significant role in the fast shift account. Instead, Schaffer collateral plasticity is subject to a continuous theta modulation, whereby LTP and a particular form of LTD (dependent on both pre- and postsynaptic firing) predominate on different phases of the theta cycle. In our model LTP and LTD occur simultaneously, and both are modulated by ACh. Second, in the "slow shift" account, mode shifting is induced by the novelty of hip- pocampal input while in the fast shift account it occurs automatically due to the differing learning and retrieval dynamics on different phases of theta. Third, as a consequence of the interplay between LTP and LTD in the model of Hasselmo et al. (2002), both old and new patterns are continuously learned, unlearned, and relearned (for new patterns LTD predominates, while for old patterns LTP and LTD balance each other out). In our model, on the other hand, most learning takes place when a pattern is new, with little learning takes place during the presentation of patterns for which a hippocampal representation already exists. Finally, although the medial septum is still assumed to pace hippocampal theta dynamics in the fast shift model, the hippocamposeptal feedback and ACh do not play a role. Hippocampal-septal interplay in that model is thus very different from that which is proposed in the present work. (One could envision a hybrid model incorporating both mechanisms, in which ACh sets broad learning dynamics and theta phase sets the balance between LTP and LTD. There would be no obvious advantage to such a combination, however.)

Several predictions distinguish between the fast and slow shift account:

1. If shifts indeed take several seconds, the effects thereof should be detectable during natural learning and memory. Specifically, learning of a new stimulus should occur quicker when an animal is in learning mode than when it is in retrieval mode (in our simulations, this difference is $200 \mathrm{~ms}$ versus $1,800 \mathrm{~ms}$, for a single, persistent EC pattern). In the "fast shift" account, there should be no detectable effect of previous stimuli on learning.

2. The slow shift account predicts that hippocampal novelty detection concurs with a drop of hippocampal activity lasting from one to a few seconds. It has, indeed, been shown that when an animal is introduced to a new environment or is presented with an unfamiliar object, this leads to a period of depressed firing of one or a few seconds in the dentate and Ammon's horn, which is followed by synchronous theta modulation. In line with our findings, this so-called "inhibitory reset" habituates with repeated presentations of the stimulus (Vinogradova et al., 1998). According to the "fast shift" account, there would be no detectable period of depressed firing.

3. Our model predicts that an interruption of hippocampal feedback to the septum would disrupt learning and retrieval in the hippocampus through disinhibition of cholinergic cells. Although some fast shift accounts may also predict this, the automatic shift incorporated in the Hasselmo et al. (2002) model would lead it to be insensitive to hippocampal feedback (unless disruption of hippocamposeptal projections turns out to affect the expression of hippocampal theta).

The first prediction lends itself to a behavioral test. It can be reformulated as: at any given moment, speed of acquisition depends on the degree of novelty of system input during the 10 or $20 \mathrm{~s}$ preceding stimulus presentation. This could be tested in an experiment with human participants, assessing continuous recognition with configurations as stimuli and short presentation times (in such experiments old and new stimuli are presented in a continuous stream; the subject has to judge which stimuli are new and which ones already appeared in the stream). A new stimulus fol- 
lowing $20 \mathrm{~s}$ of viewing old configurations should be stored less well than a stimulus appearing after one or more new stimuli.

The second prediction would most easily be tested in electrophysiological studies similar to those of Vinogradova et al. (1998), in which rats are introduced to novel and old environments while firing rates of hippocampal pyramidal cells are tracked. Our model would predict a drop of firing rate in the novel environment, as compared to the old environment, lasting many seconds longer than a fast shift account would predict. The third prediction would at the moment be difficult to test, as no procedures are available to selectively disrupt hippocampal efferents to the septum.

Our results suggest that hippocampal population activity, averaged over minutes, will not strongly distinguish between a novel and an old environment. This is because even though "activity resets" will occur more often in the novel environment, the fact that old and newly learned patterns have similar activity is likely to have a more prominent influence on this time scale. The largest distinguishing factor between the novel and old environment, on this time scale, would be ACh levels in the hippocampal formation. A recent microdialysis study supports this notion, showing that in rats, exploring a new environment, hippocampal ACh levels were considerably increased with respect to baseline, while glutamate and GABA levels were unaltered (Giovannini et al., 2001).

Other predictions generated by the simulations are not temporally specific, and thus compatible with any account that assigns a role to ACh in the storage of novel patterns:

1. Learning of a new stimulus should occur quicker in experimentally induced states of high ACh versus states of low ACh.

2. Novel configurations should lead to increased activation of cholinergic cells in the medial septum, and to a surge in hippocampal ACh release. This prediction was to some extent validated in a recent experiment showing that, during exploration of a new environment, hippocampal ACh levels were increased relative to baseline, while levels decreased during repeated exploration of the same environment (Giovannini et al., 2001).

3. Novel configurations should produce enhanced synaptic plasticity in the hippocampus proper, via effects on ACh. In as far as c-fos is an indicator of synaptic plasticity (Herdegen and Leah, 1998), the first part of this prediction gains support from a study in which rats were shown novel configurations to one eye and old ones to the other. The hippocampus contralateral to the eye viewing novel configurations expressed higher levels of c-fos than the ipsilateral hippocampus (Wan et al., 1999).

4. New patterns should elicit little activity in the hippocampus in absence of ACh. The latter prediction could be tested by subjecting rats to a novel environment, following total blockade or depletion of hippocampal $\mathrm{ACh}$, and recording from units in any or all fields of the hippocampal formation.

The model is falsified if stimulation of CA3 and CA1 does not inhibit cholinergic cells in the septum, does not influence the release of $\mathrm{ACh}$ in the hippocampus, or if any of the above predictions turn out to be untrue. In this respect it is noteworthy that the few studies addressing the function of the hippocampo-medioseptal pathway were performed in anaesthetized rats, and did not characterize the neurons responding to hippocampal input (McLennan and Miller,
1974; Dragoi et al., 1999). Moreover, the clearest effects of hippocampal influence on septal activity were observed during hippocampal sharp waves, rather than REM-associated theta (Dragoi et al., 1999). Therefore, at present there is no direct evidence for a hippocampal influence on ACh release during behavioral learning.

Another consideration regards the following: the GABAergic hippocampo-medioseptal projection appears, from anatomical studies, to terminate preferentially onto GABAergic projection neurons of the septal diagonal band complex. If this pathway were to convey the main hippocampal regulatory influence on $\mathrm{ACh}$ cells, the effect might be opposite to the one proposed by models thus far. That is, the GABAergic cells would disinhibit ACh cells in response to hippocampo-septal stimulation. Thus, ACh would rise following presentation of old patterns. However, the inhibitory hippocampo-medioseptal projection directly onto ACh neurons, albeit sparse, may be sufficient to regulate activity of ACh neurons during "mode shifting" (Dragoi et al., 1999). The combined effect of the dual hippocampo-septal pathway may be to regulate ACh activity and the strength of theta modulation in accordance with one another. Alternatively, other pathways may convey the ACh regulation according to novelty.

The present model incorporates various realistic features that are not directly related to novelty detection and mode shifting, but that enhance network performance. One of these, fast feedforward inhibition, is not commonly applied in connectionist models. Being proportional to feedforward excitation, feedforward inhibition ensures that recruitment of nodes in the target layer functions with similar selectivity over a large range of input strengths (Wierenga, 2002). The proposed function is clearly distinct from that of fast feedback inhibition, which works primarily to limit the number of recruited nodes and, therewith, the size of memory representations. In our hands this dual control of activity has proved to considerably enhance network stability, particularly in networks with recurrence amongst layers and varying pattern sizes. Notably, in the currently model the size of representations varies to some extent, depending on the modulation of system dynamics by ACh (see simulations 3 and 4).

Another interesting feature of the model is the way representations are formed in CA1. Through subthreshold inputs via the perforant path, neurons that receive a connection from active EC nodes are "predisposed" to become part of the representation. These nodes are, however, triggered by CA 3 inputs, allowing the CA1 representation to be linked to the representation in CA3 through Hebbian learning. In this way, the representation formed through self-organization in DG and CA3 can be associated to the cortical representation relayed to EC. A similar mechanism was adopted in a model by Hasselmo and Wyble (1997), although the timing of the two inputs to CA1 did not play a role in that model.

Our simulations suggest that the balance of strength between the direct perforant path and the trisynaptic input to CA1 is essential to pattern encoding. Interference with this balance, for instance through unphysiologically high levels of ACh (Fig. 8), leads to suboptimal memory performance, which might underlie the inverse U-shaped curve in dose-response curves of cholinomimetic agents in memory tasks (Wanibuchi et al., 1994).

While hippocampal feedback to the medial septum and diagonal band may set ACh levels, a novelty signal may also be conveyed to 
other targets of the hippocampus. A novelty signal over CA3 could, through the intermediaries of lateral septum, raphe nuclei, and reticular formation, influence levels of arousal (Vinogradova, 2001). In addition, a hippocampal novelty signal via the EC to the ventral striatum could influence the chain of events that leads to destabilization of thalamo-cortical representations involving the prefrontal cortex, facilitating a change of behavioral strategy. Regulation of these circuits in relation to novelty may thus instate beneficial conditions for processing and encoding of novel information.

The network presented constitutes one of the few "full-blown" models of the hippocampo-septal formation. In the present study, it was used to investigate how ACh may effectuate novelty-related modulation of hippocampal dynamics. The model may also serve as basis to explore other hypotheses, for instance regarding the significance of different parallel inputs to the hippocampus for memory processing (e.g., from different entorhinal layers, or subdivisions). It may also be evaluated how autoassociation and heteroassociation may be implemented in the circuitry; how the suppression of familiar objects in parahippocampal cortex (Xiang and Brown, 1998) affects configurational novelty detection in hippocampus, or how hippocampal subdivisions differentially contribute to neuropsychological constructs, such as recall, recognition and familiarity processing. By implementing these and other ideas in the model, consequences of assumptions about a given subdivision of the hippocampal system for other components can be traced, leading to testable predictions. In this way, computational modeling of the hippocampus can help the confrontation of theory with data, and ultimately lead to a better understanding of the structure and its functions.

\section{REFERENCES}

Acsády L, Halasy K, Freund TF. 1993. Calretinin is present in nonpyramidal cells of the rat hippocampus. III. Theri inputs from the median raphe and medial septal nuclei. Neuroscience 52:829-841.

Acsády L, Kamondi A, Sik A, Freund TF, Buzsaki G. 1998. GABAergic cells are the major postsynaptic targets of mossy fibers in the rat hippocampus. J Neurosci 18:3386-3403.

Aigner TG, Walker DL, Mishkin M. 1991. Comparison of the effects of scopolamine administered before and after acquisition in a test of visual recognition memory in monkeys. Behav Neural Biol 55:61-67.

Aloisi AM, Casamenti F, Scali C, Pepeu G, Carli G. 1997. Effects of novelty, pain and stress on hippocampal extracellular acetylcholine levels in male rats. Brain Res 748:219-226.

Alonso A, Kohler C. 1982. Evidence for separate projections of hippocampal pyramidal and non- pyramidal neurons to different parts of the septum in the rat brain. Neurosci Lett 31:209-214.

Alonso A, Klink R. 1997. Muscarinic modulation of the oscillatory and repetitive firing properties of entorhinal cortex layer II neurons. J Neurophysiol 77:1813-1828.

Amaral DG, Ishizuka N, Claiborne B. 1990. Neurons, numbers and the hippocampal network. Prog Brain Res 83:1-11.

Behrends JC, ten Bruggencate G. 1993. Cholinergic modulation of synaptic inhibition in the guinea pig hippocampus in vitro: excitation of GABAergic interneurons and inhibition of GABA-release. J Neurophysiol 69:626-629.
Benjamin AS, Bjork RA, Schwartz BL. 1998. The mismeasure of memory: when retrieval fluency is misleading as a metamnemonic index. J Exp Psychol Gen 127:55-68.

Bernardo LS, Price DA. 1982. Ionic mechanisms of cholinergic excitation in mammalian hippocampal pyramidal cells. Brain Res 249:333-344.

Bilkey DK, Goddard GV. 1985. Medial septal facilitation of hippocampal granule cell activity is mediated by inhibition of inhibitory interneurons. Brain Res 361:99-106.

Blitzer RD, Gil O, Landau EM. 1990. Cholinergic stimulation enhances long-term potentiation in the CA1 region of rat hippocampus. Neurosci Lett 119:207-210.

Bragin A, Jando G, Nadasdy Z, Hetke J, Wise K, Buzsaki G. 1995. Gamma $(40-100 \mathrm{~Hz})$ oscillation in the hippocampus of the behaving rat. J Neurosci 15:47-60.

Buhl EH, Halasy K, Somogyi P. 1994. Diverse sources of hippocampal unitary inhibitory postsynaptic potentials and the number of release sites. Nature 368:823-828.

Burgard EC, Sarvey JM. 1990. Muscarinic receptor activation facilitates the induction of long-term potentiation (LTP) in the rat dentate gyrus. Neurosci Lett 116:34-39.

Caeser M, Brown DA, Gahwiler BH, Knopfel T. 1993. Characterization of a calcium-dependent current generating a slow afterdepolarization of CA3 pyramidal cells in rat hippocampal slice cultures. Eur J Neurosci 5:560-569.

Canning KJ, Wu K, Peloquin P, Kloosterman F, Leung LS. 2000. Physiology of the entorhinal and perirhinal projections to the hippocampus studied by current source density analysis. Ann NY Acad Sci 911:5572.

Ceccarelli I, Casamenti F, Massafra C, Pepeu G, Scali C, Aloisi AM. 1999. Effects of novelty and pain on behavior and hippocampal extracellular ACh levels in male and female rats. Brain Res 815:169-176.

Chrobak JJ, Buzsaki G. 1998. Gamma oscillations in the entorhinal cortex of the freely behaving rat. J Neurosci 18:388-398.

Cobb SR, Buhl EH, Halasy K, Paulsen O, Somogyi P. 1995. Synchronisation of neuronal activity in hippocampus by individual GABAergic interneurons. Nature 378:75-78.

Cole AE, Nicoll RA. 1984. Characterization of a slow cholinergic postsynaptic potential recorded in vitro from rat hippocampal pyramidal cells. J Physiol 352:173-88.

Dickson CT, Magistretti J, Shalinski M, Hammam B, Alonso A. 2000. Oscillatory activity in entorhinal neurons and circuits: mechanisms and function. Ann NY Acad Sci 911:127-150.

Dragoi G, Carpi M, Recce M, Csicsvari J, Buzsaki G. 1999. Interactions between hippocampus and medial septum during sharp wave and theta oscillation in the behaving rat. J Neurosci 19:6191-6199.

Eichenbaum H, Dudchenko P, Wood E, Shapiro M, Tanila H. 1999. The hippocampus, memory and place cells: is it a spatial memory or a memory space? Neuron 23:209-226.

Fanselow MS. 2000. Contextual fear, gestalt memories, and the hippocampus. Behav Brain Res 110:73-81.

Freund T, Antal M. 1988. GABA-containing neurons in the septum control inhibitory interneurons in the hippocampus. Nature 336:170-173.

Frotscher M, Leranth C. 1985. Cholinergic innervation of the hippocampus as revealed by choline acetyltransferase immunocytochemistry: a combined light and electron microscopic study. J Comp Neurol 239: 237-246.

Gardiner JM, Craik FIM, Bleasdale FA. 1973. Retrieval difficulty and subsequent recall. Mem Cognit 1:213-216.

Ghoneim MM, Mewaldt SP. 1975. Effects of diazepam and scopolamine on storage retrieval and organization processes in memory. Psychopharmacologia 44:257-262.

Giovannini MG, Rakovska A, Benton RS, Pazzagli M, Bianchi L, Pepeu G. 2001. Effects of novelty and habituation on acetylcholine, GABA, and glutamate release from the frontal cortex and hippocampus of freely moving rats. Neuroscience 106:43-53. 
Green JD, Arduini AA. 1954. Hippocampal electrical activity in arousal. J Neurophysiol 17:533-557.

Grossberg S. 1976. Adaptive pattern classification and universal recoding. II. Feedback, expectation, olfaction, and illusions. Biol Cybern 23: 187-202.

Gulyás AI, Görcs TJ, Freund TF. 1990. Innervation of different peptidecontaining neurons in the hippocampus by GABAergic septal afferents. Neuroscience 37:31-44.

Gulyás A, Seress L, Tóth K, Acsady L, Antal M, Freund T. 1991. Septal GABAergic neurons innervate inhibitory interneurons in the hippocampus of the macaque monkey. Neuroscience 41:381-390.

Hasselmo ME. 1995. Neuromodulation and cortical function: modeling the physiological basis of behavior. Behav Brain Res 67:1-27.

Hasselmo ME, Bower JM. 1993. Acetylcholine and memory. Trends Neurosci 16:218-222.

Hasselmo ME, Fehlau P. 2001. Differences in time course of ACh and GABA modulation of excitatory synaptic potentials in slices of rat hippocampus. J Neurophysiol 86:1792-1802.

Hasselmo ME, Schnell E. 1994. Laminar selectivity of the cholinergic suppression of synaptic transmission in rat hippocampal region CA1: computational modeling and brain slice physiology. J Neurosci 14: 3898-3914.

Hasselmo ME, Wyble BP. 1997. Free recall and recognition in a network model of the hippocampus: simulating effects of scopolamine on human memory function. Behav Brain Res 89:1-34.

Hasselmo ME, Schnell E, Barkai E. 1995. Dynamics of learning and recall at excitatory recurrent synapses and cholinergic modulation in rat hippocampal region CA3. J Neurosci 15(7 Pt 2):5249-5262.

Hasselmo ME, Wyble BP, Wallenstein GV. 1996. Encoding and retrieval of episodic memories: role of cholinergic and GABAergic modulation in the hippocampus. Hippocampus 6:693-708.

Hasselmo ME, Bodelón C, Wyble BP. 2002. A proposed function for hippocampal theta rhythm: separate phases of encoding and retrieval enhance reversal of prior learning. Neural Comput 14:793-817.

Henze DA, Urban NN, Barrionuevo G. 2000. The multifarious hippocampal mossy fiber pathway: a review. Neuroscience 98:407-427.

Herdegen T, Leah JD. 1998. Inducible and constitutive transcription factors in the mammalian nervous system: control of gene expression by Jun, Fos and Krox, and CREB/ATF proteins. Brain Res Rev 28: $370-490$.

Hodgkin AL, Huxley AF. 1952. A quantitative description of ion currents and its application to conduction and excitation in nerve membranes. J Physiol 117:500-544.

Holscher C, Anwyl R, Rowan MJ. 1997. Stimulation on the positive phase of hippocampal theta rhythm induces long-term potentiation that can Be depotentiated by stimulation on the negative phase in area CA1 in vivo. J Neurosci 17:6470-6477.

Hounsgaard J. 1978. Presynaptic inhibitory action of acetylcholine in area CA1 of the hippocampus. Exp Neurol 62:787-797.

Huerta PT, Lisman JE. 1993. Heightened synaptic plasticity of hippocampal CA1 neurons during a cholinergically induced rhythmic state. Nature 364:723-725.

Huerta PT, Lisman JE. 1995. Bidirectional synaptic plasticity induced by a single burst during cholinergic theta oscillation in CA1 in vitro. Neuron 15:1053-1063.

Johnson LL, Moberg GP. 1980. Adrenocortical response to novelty stress in rats with dentate gyrus lesions. Neuroendocrinology 30:187-192.

Kitchigina V, Vankov A, Harley C, Sara SJ. 1997. Novelty-elicited, noradrenaline-dependent enhancement of excitability in the dentate gyrus. Eur J Neurosci 9:41-47.

Knight RT, Nakada T. 1998. Cortico-limbic circuits and novelty: a review of EEG and blood flow data. Rev Neurosci 9:57-70.

Kopelman MD. 1986. The cholinergic neurotransmitter system in human memory and dementia: a review. Q J Exp Psychol 38A:535-573.

Larson J, Lynch G. 1986. Induction of synaptic potentiation in hippocampus by patterned stimulation involves two events. Science 232: 985-988.
Lee MA, Jayathilake K, Meltzer HY. 1999. A comparison of the effect of clozapine with typical neuroleptics on cognitive function in neuroleptic-responsive schizophrenia. Schizophr Res 37:1-11.

Lemaire V, Aurousseau C, Le Moal M, Abrous DN. 1999. Behavioural trait of reactivity to novelty is related to hippocampal neurogenesis. Eur J Neurosci 11:4006-4014.

Levy WB. 1996. A sequence predicting CA3 is a flexible associator that learns and uses context to solve hippocampal-like tasks. Hippocampus 6:579-590.

Li XG, Somogyi P, Ylinen A, Buzsaki G. 1994. The hippocampal CA3 network: an in vivo intracellular labeling study. J Comp Neurol 339: 181-208.

Lisman JE. 1999. Relating hippocampal circuitry to function: recall of memory sequences by reciprocal dentate-CA3 interactions. Neuron 22:233-242.

Lisman JE, Otmakhova NA. 2001. Storage, recall, and novelty detection of sequences by the hippocampus: elaborating on the SOCRATIC model to account for normal and aberrant effects of dopamine. Hippocampus 11:551-568.

MacGregor RJ, Oliver RM. 1974. A model for repetitive firing in neurons. Cybernetik 16:53-64.

Madison DV, Nicoll RA. 1984. Control of the repetitive discharge of rat CA 1 pyramidal neurones in vitro. J Physiol 354: 319-331.

Madison DV, Lancaster B, Nicoll RA. 1987. Voltage clamp analysis of cholinergic action in the hippocampus. J Neurosci 7:733-741.

Malmberg KJ, Shiffrin RM. 2003. The "one-shot" hypothesis and the list-strength effect for free recall. J Exp Psychol Learn Mem Cogn (in press).

Marr D. 1971. Simple memory: a theory for archicortex. Phil Trans R Soc Lond B 262:23-81.

McClelland JL, Goddard GV. 1996. Considerations arising from a complementary learning systems perspective on hippocampus and neocortex. Hippocampus 6:654-665.

McClelland JL, McNaughton BL, O’Reilly RC. 1995. Why there are complementary learning systems in the hippocampus and neocortex: insights from the successes and failures of connectionist models of learning and memory. Psychol Rev 102:419-457.

McLennan H, Miller JJ. 1974. The hippocampal control of neuronal discharges in the septum of the rat. J Physiol 237:607-624.

McNaughton BL, Morris RGM. 1987. Hippocampal synaptic enhancement and information storage within a distributed memory system. Trends Neurosci 10:408-415.

Meeter M, Murre JMJ, Talamini LM. 2002. A computational approach to memory deficits in schizophrenia. Neurocomputing 44:929-936.

Mewaldt SP, Ghoneim MM. 1979. The effect and interactions of scopolamine, physostigmine and methamphetamine on human memory. Pharmacol Biochem Behav 10:1205-1210.

Miles R, Tóth K, Gulyás AI, Hajos N, Freund TF. 1996. Differences between somatic and dendritic inhibition in the hippocampus. Neuron 6:815-823.

Montag-Sallaz M, Welzl H, Kuhl D, Montag D, Schachner M. 1999. Novelty-induced increased expression of immediate-early genes c-fos and arg 3.1 in the mouse brain. J Neurobiol 38:234-246.

Mumby DG, Gaskin S, Glenn MJ, Schramek TE, Lehmann H. 2002. Hippocampal damage and exploratory preferences in rats: memory for objects, places, and contexts. Learn Mem 9:49-57.

Murdock BB Jr. 1974. Human memory: theory and data. Hillsdale, NJ: Lawrence Erlbaum.

Murre JMJ. 1992. Categorization and learning in modular neural networks. Hillsdale, NJ: Lawrence Erlbaum.

Myers CE, Gluck MA. 1994. Context, conditioning and hippocampal re-representation. Behav Neurosci 108:835-847.

Nadel L, Samsonovitch A, Ryan L, Moscovitch M. 2000. Multiple trace theory of human memory: computational, neuroimaging and neuropsychological results. Hippocampus 10:352-368.

O'Keefe J, Nadel L. 1978. The hippocampus as a cognitive map. Oxford: Clarendon Press. 
O’Keefe J, Recce ML. 1993. Phase relationship between hippocampal place units and the EEG theta rhythm. Hippocampus 3:317-330.

O'Reilly RC, McClelland JL. 1994. Hippocampal conjunctive encoding, storage, and recall: avoiding a trade-off. Hippocampus 4:661682.

Orsetti M, Casamenti F, Pepeu G. 1996. Enhanced acetylcholine release in the hippocampus and cortex during acquisition of an operant behavior. Brain Res 724:89-96.

Peterson RC. 1977. Scopolamine induced learning failures in man. Psychopharmacology 52:283-289.

Potter MC. 1976. Short-term conceptual memory for pictures. J Exp Psychol Hum Learn Mem 2:509-522.

Roberts WA. 1972. Free recall of word lists varying in length and rate of presentation: a test of total-time hypotheses. J Exp Psychol Gen 92: 365-372.

Rolls ET. 1996. A theory of hippocampal function in memory. Hippocampus 6:601-620.

Rudy JW, Barrientos RM, O’Reilly RC. 2002. Hippocampal formation supports conditioning to memory of a context. Behav Neurosci 116 : $530-538$.

Scharfman HE. 1996. Conditions required for polysynaptic excitation of dentate granule cells by area CA3 pyramidal cells in rat hippocampal slices. Neuroscience 72:655-668.

Shimono K, Brucher F, Granger R, Lynch G, Taketani M. 2000. Origins and distribution of cholinergically induced beta rhythms in hippocampal slices. J Neurosci 20:8462-8473.

Sik A, Penttonen M, Ylinen A, Buzsaki G. 1995. Hippocampal CA1 interneurons: an in vivo intracellular labeling study. J Neurosci 15: 6651-6665.

Skaggs WE, McNaughton BL, Wilson MA, Barnes CA. 1996. Theta phase precession in hippocampal neuronal populations and the compression of temporal sequences. Hippocampus 6:149-172.

Sohal VS, Hasselmo ME. 1998. Gaba-b modulation improves sequence disambiguation in computational models of hippocampal region CA3. Hippocampus 8:171-193.

Soltez I, Deschenes M. 1993. Low and high-frequency membrane potential oscillations during theta activity in CA1 and CA3 pyramidal neurons of the rat hippocampus under ketamine-xylazine anesthesia. J Neurophysiol 70:97-116.

Squire LR. 1992. Memory and the hippocampus: a synthesis from findings with rats, monkeys, and humans. Psychol Rev 99:195-231.

Squire LR, Alvarez P. 1995. Retrograde amnesia and memory consolidation: a neurobiological perspective. Curr Opin Neurobiol 5:169-175.

Stewart M, Fox SE. 1989a. Detection of an atropine-resistant component of the hippocampal theta rhythm in urethane-anesthetized rats. Brain Res 500:55-60.

Stewart M, Fox SE. 1989b. Two populations of rhythmically bursting neurons in rat medial septum are revealed by atropine. J Neurophysiol 61:982-993.

Stewart M, Fox SE. 1990. Do septal neurons pace the hippocampal theta rhythm? Trends Neurosci 13:163-168.

Suzuki WA. 2000. The neurophysiology of memory. Ann NY Acad Sci 911:175-191.

\section{APPENDIX}

Integrate-and-fire MacGregor model neurons were used for the model. In running the simulations, the discrete-time approximation formulas given by MacGregor and Oliver (1974) were used. The model was constructed using the Nutshell simulator, devel-
Tóth K, Freund TF. 1992. Calbindin D28k-containing nonpyramidal cells in the rat hippocampus: their immunoreactivity for GABA and projection to the medial septum. Neuroscience 49:793-805.

Tóth K, Borhegyi Z, Freund TF. 1993. Postsynaptic targets of GABAergic hippocampal neurons in the medial septum-diagonal band of Broca complex. J Neurosci 13:3712-3724.

Tóth K, Freund TF, Miles R. 1997. Disinhibition of rat hippocampal pyramidal cells by GABAergic afferents from the septum. J Physiol 500:463-474.

Traub RD, Spruston N, Soltesz I, Konnerth A. 1998. Gamma-frequency oscillations: a neuronal population phenomenon, regulated by synaptic and intrinsic cellular processes, and inducing synaptic plasticity. Prog Neurobiol 55:563-575.

Treves A, Rolls ET. 1994. Computational analysis of the role of the hippocampus in memory. Hippocampus 4:374-391.

Vinogradova OS. 2001. Hippocampus as comparator: role of the two input and two output systems of the hippocampus in selection and registration of information. Hippocampus 11:578-598.

Vinogradova OS, Kitchigina VF, Zenchenko CI. 1998. Pacemaker neurons of the forebrain medial septal area and theta rhythm of the hippocampus. Membr Cell Biol 11:715-725.

Wan H, Aggleton JP, Brown MW. 1999. Different contributions of the hippocampus and perirhinal cortex to recognition memory. J Neurosci 19:1142-1148.

Wanibuchi F, Nishida T, Yamashita H, Hidaka K, Koshiya K, Tsukamoto S, Usuda S. 1994. Characterization of a novel muscarinic receptor agonist, YM796: comparison with cholinesterase inhibitors in in vivo pharmacological studies. Eur J Pharmacol 265:151-158.

Whittington MA, Doheny HC, Traub RD, LeBeau FE, Buhl EH. 2001. Differential expression of synaptic and non-synaptic mechanisms underlying stimulus-induced gamma oscillations in vitro. J Neurosci 21: 1727-1738.

Wierenga C. 2002. Functional interactions between interneurons and the pyramidal cell population in the hippocampal CA1 area. Swammerdam Institute for Life Sciences. Amsterdam: University of Amsterdam.

Whishaw IQ. 1989. Dissociating performance and learning deficits on spatial navigation tasks in rats subjected to cholinergic muscarinic blockade. Brain Res Bull 23:347-358.

Witter MP, Wouterlood FG, Naber PA, Van Haeften T. 2000. Anatomical organization of the parahippocampal-hippocampal network. Ann NY Acad Sci 911:1-24.

Xiang JZ, Brown, MW. 1998. Differential neuronal encoding of novelty, familiarity, and recency in regions of the anterior temporal lobe. Neuropharmacology 37:657-676.

Yeckel MF, Berger TW. 1990. Feedforward excitation of the hippocampus by afferents from the entorhinal cortex: redefinition of the role of the trisynaptic pathway. Proc Natl Acad Sci USA 87: 5832-5836.

Ylinen A, Soltesz I, Bragin A, Penttonen M, Sik A, Buzsaki G. 1995. Intracellular correlates of hippocampal theta rhythm in identified pyramidal cells, granule cells and basket cells. Hippocampus 5:78-90.

Zhu XO, McCabe BJ, Aggleton JP, Brown MW. 1997. Differential activation of the rat hippocampus and perirhinal cortex by novel visual stimuli and a novel environment. Neurosci Lett 229:141-143.

oped by our group. It can be downloaded, without cost, at www. neuromod.org/nutshell.

MacGregor and Oliver (1974) derived their model neuron from the Hodgkin-Huxley formulas (Hodgkin and Huxley, 1952) to account for firing characteristics in single neurons, while being computationally inexpensive enough for use in large-scale networks. These model neurons show spiking, adaptation, and threshold accommodation (accommodation was not implemented in the 
present simulations). They are updated in discrete time steps, which in our simulations lasted 2 ms.

The model neuron emits a spike every time the membrane potential $E$ crosses the threshold $\theta$ :

$$
E \geq \theta \Rightarrow S=1
$$

In equation $1, S$ is a dichotomous variable that is equal to 1 if the node emits a spike, and equals 0 otherwise. The membrane potential, $E$, is dependent on the sodium, potassium, and chloride currents over the membrane, as described in the following differential equation:

$$
\frac{d E}{d t}=-\delta E-g_{k}\left(E-E_{k}\right)-g_{e x}\left(E-E_{e x}\right)-g_{i}\left(E-E_{i}\right)-S E
$$

Here, $-\delta E$ is the leak current, $g_{\text {ex }}$ the excitatory conductance, $E_{e x}$ is the sodium reversal potential, $g_{i}$ is the inhibitory conductance, and $E_{i}$ is the chloride reversal potential. For computational purposes, both the membrane potential and the reversal potentials were mapped onto the interval $[-1,7]$ via a simple linear transformation (MacGregor and Oliver, 1974). Resulting potential is equated to $0(-75 \mathrm{mV})$, the firing threshold $\theta$ to $1(-60 \mathrm{mV})$, the sodium reversal potential to $7(+30 \mathrm{mV})$, and both the potassium and chloride reversal potentials to $-1(-90 \mathrm{mV})$. The parameter governing the leak current, $\delta$, is set to $1 / 7$. When the node emits a spike, membrane potential is reset to resting level (via the term $S E$ ).

The potassium conductance $g_{k}$ models adaptation and is determined by

$$
\frac{d g_{k}}{d t}=-g_{k} / \tau+b S
$$

where $S$ is the spiking variable. The time constant $\tau$ is set to $1 / 13$, the gain parameter $b$ to 0.35 . Excitatory input to the $i$ th node is a simple linear summation of weighted inputs to that node:

$$
g_{\text {ex }}=\sum_{j} w_{i j} S_{j}
$$

where $w_{i j}$ is the weight from node $j$ to node $i$, and $S_{j}$ is the spiking variable of node $j$. Rise times of synaptic inputs are thus not taken into account.

Simple Hebbian learning is used, modeling LTP, with the additions of negative Hebbian learning, modeling LTD, and a bound on connection weights. Weights are changed according to

$$
\Delta w_{i j}=\mu^{+} S_{i} S_{j}-\mu^{-} S_{i}\left(1-S_{j}\right)
$$

Here, $w_{i j}$ is the weight from node $j$ to node $i$, while $S_{i}$ and $S_{j}$ are the spiking variables of the receiving and sending node, respectively. This is subject to the constraints that a weight cannot be lower than 0 or exceed a maximum $W$. The positive learning rate, $\mu^{+}$, as well as the maximum weight, $W$, are set separately for every connection (Table 2). The negative learning rate $\mu^{-}$is set to $75 \%$ of the positive learning rate in all connections.

The inhibitory conductance, $g_{i}$, in a given layer, $l$, is modeled as a continuous variable reflecting firing rates of inhibitory interneurons. It is described by the following equation:

$$
g_{i t}^{l}=1+i_{t}^{l}-s_{t}
$$

where $s_{t}$ is the activity of the septal interneuron:

$$
s_{t}=0.5-0.5 \sin (t / f)
$$

This is a simple sinusoid between 0 and 1 with a frequency of $f$ (set to 50, equivalent to a 200-ms $\theta$-band oscillation). The other component of Equation 6, $i^{1}$, models the activity of intrinsic interneurons:

$$
i_{t}^{l}=\alpha_{i} i_{t-1}^{l}+\beta^{l}\left(A_{t-1}^{l}\right)+\sum_{p} \lambda^{l p} A_{t-1}^{p}
$$

Thus, inhibition in layer $l$ on time step $t$ is a function of the feedforward and feedback activation of inhibitory cells by the pyramidal cells, and of inhibition on time step $t-1$. Feedforward and feedback inhibition are linear functions of the excitatory activation in the layers connecting to layer $l$ (feedforward), and of excitatory activation in layer $l$ itself (feedback). The activation of each layer $\left(A^{1}\right)$ is calculated by dividing the number of firing nodes in the layer by its maximum $k^{1}$ (Table 1 ). The $\beta^{l}$ parameters (strength of feedback inhibition to layer $l$ ) in all layers are given in Table 1 , the $\lambda^{l p}$ parameters associated with each connection (strength of feedforward inhibition from layer $p$ to layer $l$ ) are listed in Table 2. No rise time is included in the formula for inhibition, as our 2-ms time step made this redundant. However, the decay parameter of the current $\left(\alpha_{i}\right)$ was set by fitting a single exponential to the double exponential used by Sohal and Hasselmo (1998); $\alpha_{i}=0.76$.

In very large networks, the inhibition described above is sufficient to constrain activity. In networks of the size used here, however, random fluctuations may produce large swings in activity that can be kept in check with a fast cutoff mechanism. This mechanism allows no more than a $k^{1}$ number of nodes to fire in a layer at any given time step (Table 1 ). If more than $k^{1}$ nodes cross the firing threshold, only the $k^{1}$ nodes with the highest membrane potential are allowed to fire.

ACh levels in the model are regulated by inhibitory activity in layers CA3 and CA1. Activity of the septal cholinergic neurons, $A_{t}^{s}$, is set to $F$-inhibition (see Eq. 9). Here, $F$, set to 1 in all simulations, represents excitation of the septum by sources external to the model, such as the reticular formation. Inhibition comes from the septal oscillator interneurons, $s_{t}$ (whose output is the $\theta$-frequency sinusoid given by Eq. 7), and from the hippocampal afferents, $i_{t}^{\text {s. A }}$ moving average of inhibition in CA 1 and CA 3 determines $i_{t}^{s}$ (given by Eq. 10):

$$
A_{t}^{s}=F-s_{t}-i_{t}^{s} \quad \text { if } \quad F-s_{t}-i_{t}^{s} \geq 0 \quad \text { else } \quad A_{t}^{s}=0
$$

$$
i_{t}^{s}=\alpha^{s} i_{t-1}^{s}+\beta^{s}\left(i_{t-1}^{C A 3}+i_{t-1}^{C A 1}\right)
$$

The parameter $\alpha^{s}$ is set to 0.85 , and $\beta^{s}$ to 0.45 . Release of ACh is equal to the activity of the septal cholinergic node, $A_{t}^{s}$. This release, in turn, determines ACh modulation in the hippocampus, for which we use the symbol $\Psi_{t}$, following Hasselmo and colleagues (e.g., Hasselmo et al., 1995). At each time step, the amount of ACh released is fed into a dual exponential:

$$
\Psi_{t}=\sum_{d} A_{d}^{s}\left(e^{-\tau_{1}(t-d)}-e^{-\tau_{2}(t-d)}\right)
$$


The time constants $\left(\tau_{1}, \tau_{2}\right)$ of the dual exponential were rescaled from those found by Hasselmo and Fehlau (2001), who fitted a dual exponential to experimental data on the time course of ACh modulation data $\left(\tau_{1}=0.001258, \tau_{2}=0.00015\right)$. These values correspond to a slow rise, with a maximum at $\sim 3.5 \mathrm{~s}$, and a decrease back to 0 in 10 to $20 \mathrm{~s}$.

As the effects of ACh have been discussed in the main text, only their implementation are listed:

1. For preferential dampening of transmission over Schaffer collaterals to CA3 and CA1, transmission in these two tracts $\left(g_{e x}\right.$ in Eq. 4) is multiplied by a factor $1-0.6^{*} \Psi$.
2. For enhancement of LTP at CA3 recurrent collateral synapses and at CA1 Schaffer collateral synapses, the learning rate ( $\mu$ in Eq. 5 ) is multiplied by $\Psi$ in these connections.

3. Reduction of firing adaptation of DG, CA3, and CA1 excitatory cells is effectuated by multiplication of the adaptation constant ( $b$ in Eq. 3) with a factor $1-\Psi$.

4. Suppression of inhibition in all model layers is achieved multiplying the feedback inhibition constant ( $\alpha$ in Eq. 8 ) by a factor $1-0.5^{*} \Psi$.

5. A mild depolarization of DG, CA3, and CA1 principle cells is implemented adding a constant factor, $0.12^{*} \Psi$, to the input of cells in these layers ( $g_{e x}$ in Eq. 4). 